\title{
Some Independence Results for Control Structures in Complete Numberings
}

\author{
Sanjay Jain \\ School of Computing \\ National University of Singapore \\ Singapore 119260, Rep. of Singapore \\ sanjay@comp.nus.edu.sg \\ Jochen Nessel \\ FB Informatik \\ Universität Kaiserslautern \\ Postfach 3049, 67653 Kaiserslautern \\ Fed. Rep. of Germany \\ nessel@informatik.uni-kl.de
}

March 11, 2007

\begin{abstract}
Acceptable programming systems have many nice properties like s-m-n-Theorem, Composition and Kleene Recursion Theorem. Those properties are sometimes called control structures, to emphasize that they yield tools to implement programs in programming systems. It has been studied, among others by Riccardi and Royer, how these control structures influence or even characterize the notion of acceptable programming system. The following is an investigation, how these control structures behave in the more general setting of complete numberings as defined by Mal'cev and Eršov.
\end{abstract}




\section{Introduction}

The notion of acceptable numbering (also called acceptable programming system) describes the usual programming languages like Pascal, C, Fortran and the like, and provides an elegant tool to prove results about this kind of languages in a universal and abstract setting.

Acceptable numberings have been characterized in various ways; see below for references. Mostly by saying that a numbering is acceptable if and only if it enumerates every partial recursive function and has an additional property, for example it satisfies the s-m-n-Theorem. The s-m-n-Theorem and other similar properties can be understood as effective means to build programs in a numbering or - equivalently - programming system. So they were termed control structures by Riccardi and Royer.

Beside acceptable numberings, also numberings for subclasses of the partial recursive functions have been studied. In this paper we will investigate the notion of complete numbering as defined by Mal'cev and Eršov and the stronger notion of $U$-acceptable numbering. Intuitively, this kind of numberings should model programming systems that do not contain every partial recursive function, but come as close as possible to the "programming comfort" of acceptable numberings. For example, although a programming system does not contain every partial recursive function, one might want to have nice programming tools such as the s-m-n-Theorem, composition or the Kleene Recursion Theorem to ease work within the system.

The programming comfort of a programming system is usually measured by the user in terms of the control structures it provides: the more and powerful the structures, the better the system. In acceptable numberings, many control structures can be shown to be equivalent, i.e., one can be obtained from the other. Moreover, the acceptable numberings can be characterized using control structures. The following investigates how those control structures behave in the more general setting of complete and $U$-acceptable numberings. As it will turn out, most of the considered control structures will be independent, i.e., one can not be "simulated" by the other. This means, for programming systems not containing every partial recursive function, one has to be careful to design into the system whatever control structures are desired. Furthermore the results indicate, that it is not single control structures that characterize the notions of completeness and $U$-acceptability, in contrast to acceptable numberings; cf. Theorem 1. It would be interesting to know then, what properties could be employed to characterize complete 
and $U$-acceptable numberings.

Applications for numberings not containing every partial recursive functions can be found, for example, in machine learning. Learning success often depends on the choice of a suitable programming system which is then used as a hypothesis space. In many occasions those suitable hypothesis spaces, i.e., the underlying programming systems, are not acceptable programming systems; see $[1,3,9,10]$ among others. The work in $[9,10]$ on language learning often uses hypothesis spaces containing only total recursive predicates, which are interpreted as languages. Those results often depend on whether a hypothesis space may contain only the languages to be learned or if the space contains more languages: redundant spaces often give greater learning power. [3] also deals with language learning. The goal there was to find control structures that, if present in a numbering representing a hypothesis space, ensure learnability within this space. It contains a theorem stating that a numbering is "limiting acceptable" if and only if every languages class, that is learnable in the limit, is learnable using this numbering as (implicit) hypothesis space and if the numbering has a control structure called 'limiting projection' - we refer the interested reader to the mentioned publication for definitions and details.

Two of the purposes of the present article are, to provide tools to achieve the goals of [3] and to gain better understanding of the phenomena observed in $[9,10]$.

Other results involving non-acceptable numberings are characterizations of learnable classes of total recursive functions. They usually have the form "a class of total recursive functions is learnable 'with respect to some criteria' if and only if there exists a numbering with certain properties"; cf. for example $[5,6,8]$. The techniques employed in this paper could now be used to investigate how comfortable or "user friendly" those numberings are. Or if it is possible to add additional control structures to the used numberings without the structures interfering with learnability. Since, for example, each recursively enumerable - see below for definition - class of total recursive is learnable in the limit, having "comfortable" hypothesis spaces might be valuable for potential users, in order to work with results obtained by learning algorithms within such a rather big hypothesis space.

The paper is organized in the following way. In section 2 we give definitions and a short survey over previous work by Mal'cev and Eršov. Section 3 is devoted to the proof that the notion of $U$-acceptable numbering is more restrictive than the notion of $U$-complete numbering. In section 4 we provide 
the independence results for control structures in complete numberings.

\section{Definitions and previous work}

The following text assumes familiarity with standard mathematical and recursion theoretic notions and notations as given for example in [15]. For $A \subseteq \mathbb{N}$, let $\max (A)$ and $\min (A)$ stand for the maximum and minimum value in $A$. We define $\max (\emptyset)=0$ and $\min (\emptyset)=\infty$.

$\langle\cdot, \cdot\rangle$ denotes a computable, one-to-one, and onto pairing function from pairs of natural numbers to natural numbers. By $\pi_{1}$ and $\pi_{2}$ we denote the corresponding projection functions. This pairing function can be naturally extended to encoding of triples etc. (for example, one can take $\langle x, y, z\rangle=$ $\langle x,\langle y, z\rangle\rangle)$.

In the following, $f, g, h$ range over (possibly partial) functions; domain $(f)$ and range $(f)$ denote the domain and range of a function $f$, respectively. By $f \circ g$ we denote the composition of $f$ and $g$. For functions $f, g, f \subseteq g$ means that $f$ is a subfunction of $g$. We write $f(x) \downarrow$ to denote that $f$ is defined on $x$ and $f(x) \uparrow$ to denote that $f$ is undefined on $x$. We often identify functions with their graphs. Thus, the everywhere undefined function may also be written $\emptyset$. $F_{0}, F_{1}, F_{2}, \ldots$, denotes a canonical recursive enumeration of all and only the finite functions, where one can effectively determine $\operatorname{card}\left(\operatorname{domain}\left(F_{i}\right)\right)$ and graph of $F_{i}$ from $i$; cf. [15].

A set of functions $U$ is said to have a least element if there exists an element $\perp \in U$ such that for all $f \in U, \perp \subseteq f$. Note that if $U$ has a least element, then this least element is unique.

For $n \geq 1, \mathcal{R}^{n}$ and $\mathcal{P}^{n}$ denote the sets of all total and partial recursive functions of $n$ arguments, respectively. We define $\mathcal{R}=\mathcal{R}^{1}$ and $\mathcal{P}=\mathcal{P}^{1}$.

Any function from $\mathcal{P}^{2}$ is called a computable numbering (also called a programming system). Since we will only be concerned about computable numberings, in this paper we often drop "computable" from "computable numbering". From now on, $\psi, \eta, \zeta$ and the like range over numberings. For a numbering $\psi, \psi_{i}$ means $\lambda x . \psi(i, x)$, and $i$ is said to be an index or program for the function $\psi_{i}$. Furthermore, $\mathcal{P}_{\psi}=\left\{\psi_{i} \mid i \in \mathbb{N}\right\}$ is the set of partial recursive functions enumerated by the numbering $\psi$. If $U=\mathcal{P}_{\psi}$, we say that $\psi$ is a numbering for $U$. If $\mathcal{P}_{\eta} \subseteq \mathcal{P}_{\psi}$ for numberings $\eta$ and $\psi$, then $\eta$ is called a subnumbering of $\psi . U \subseteq \mathcal{P}$ is called recursively enumerable, if there exists $\psi$ such that $U=\mathcal{P}_{\psi}$. Suppose $\Psi$ is a fixed Blum complexity measure [2] for 
numbering $\psi$. Then $\psi_{i, s}$ denotes the function

$$
\psi_{i, s}(x)= \begin{cases}\psi_{i}(x), & \text { if } x<s \text { and } \Psi_{i}(x)<s \\ \uparrow, & \text { otherwise. }\end{cases}
$$

The index set for a function $f$ in the numbering $\psi$, denoted by $I_{f}^{\psi}$, is $I_{f}^{\psi}=\left\{i \mid \psi_{i}=f\right\}$. This notion of index sets can be extended to sets $V$ of functions: $I_{V}^{\psi}=\bigcup_{f \in V} I_{f}^{\psi}$.

A numbering $\eta$ is said to be reducible to a numbering $\psi$, abbreviated $\eta \preceq$ $\psi$, if there is a recursive function $r$ such that $\eta_{i}=\psi_{r(i)}$ for all $i$. Note that $\preceq$ is reflexive and transitive. If $\eta$ is reducible to $\psi$ via recursive function $r$, then we denote this fact by $\eta \preceq_{r} \psi$. Two numberings $\psi, \eta$ are called equivalent if $\psi \preceq \eta$ and $\eta \preceq \psi$, and isomorphic if they are equivalent by a one-to-one and onto recursive function. A numbering $\psi$ is said to be acceptable, if $\eta \preceq \psi$ for all numberings $\eta$. By $\varphi$ we denote a standard acceptable numbering. By $\Phi$ we denote a fixed Blum complexity measure for $\varphi$.

Intuitively, the most important numberings are the acceptable ones. One of the reasons is that most natural programming languages are in fact acceptable numberings. They have many nice properties, including

- universality, i.e., every partial recursive function appears within the numbering,

- composition, i.e., any two programs can be effectively combined into a third one,

- s-m-n-Theorem, i.e., input variables can be made constants within the program,

- recursion theorems, like the Kleene Recursion Theorem (KRT) and the Rogers Fixed Point Theorem (FPT), which make possible proofs using self-referential arguments.

The following is a more formal definition of what it means for a numbering to "have" composition or some other properties. If in the rest of the paper $\psi$ is said "to have some property" or to "satisfy a theorem" not defined below, it should be interpreted analogously.

Definition 1 Let $\psi$ be any numbering. 
(a) $\psi$ has composition if there exists comp $\in \mathcal{R}^{2}$ such that $\psi_{\text {comp }(i, j)}(x)=$ $\psi_{i}\left(\psi_{j}(x)\right)$ for all $i, j, x$.

(b) $\psi$ satisfies (effective) $s$-m-n-Theorem if there exists $s \in \mathcal{R}^{2}$ such that $\psi_{s(x, y)}(z)=\psi_{x}(\langle y, z\rangle)$ for all $x, y, z$. We will say that $\psi$ satisfies non-effective s-m-n-Theorem to emphasize the fact that the function $s$ exists, but it might not be computable. So effective s-m-n-Theorem immediately yields noneffective s-m-n-Theorem. If not otherwise stated, effective s-m-n-Theorem is assumed.

(c) $\psi$ has infinite padding if there exists $p \in \mathcal{R}$ such that, for all $i, \psi_{p(i)}=$ $\psi_{i}$, and $p(i)>i$.

(d) $\psi$ satisfies (non-effective) KRT (Kleene Recursion Theorem) if for every $i$ there exists an $e$ such that $\psi_{e}(x)=\psi_{i}(\langle e, x\rangle)$, for all $x$. If nothing else is stated, non-effective KRT is assumed.

(e) $\psi$ satisfies effective KRT if there exists $r \in \mathcal{R}$ such that $\psi_{r(i)}(x)=$ $\psi_{i}(\langle r(i), x\rangle)$, for all $i, x$.

(f) $\psi$ satisfies FPT (Fixed Point Theorem) if for every $h \in \mathcal{R}$ there exists $n$ such that $\psi_{n}=\psi_{h(n)}$. If $\psi_{h(n)}=\psi_{n}$, then we call $n$ the fixed point of $\psi$ for $h$.

(g) $\psi$ satisfies $R T$ (Rice's Theorem) if for every $U \subseteq \mathcal{P}_{\psi}, I_{U}^{\psi}$ is recursive if and only if $U=\emptyset$ or $U=\mathcal{P}_{\psi}$.

Acceptable numberings have been characterized in various ways, the following theorem only lists a few examples.

\section{Theorem 1}

Let $\psi$ be any numbering. Then the following statements are equivalent.

(a) $\psi$ is acceptable.

(b) $\mathcal{P}_{\psi}=\mathcal{P}$ and $\psi$ has composition.

(c) $\mathcal{P}_{\psi}=\mathcal{P}$ and $\psi$ satisfies $s$-m-n-Theorem.

(d) $\mathcal{P}_{\psi}=\mathcal{P}$ and $\psi$ satisfies Parametric Recursion Theorem (see below).

(e) $\mathcal{P}_{\psi}=\mathcal{P}$ and $\psi$ satisfies effective KRT and has infinite padding.

Proof. See $[11,15,13]$.

Properties like the ones just defined are called control structures in the work of Riccardi and Royer, cf. [13, 16], though their representation is different. The intuition behind this denotation is, that composition and sm-n-Theorem give "programming tools" for numberings, i.e., means to build 
programs. For more motivation, the interested reader is referred to the above mentioned publications.

Now we give the definition of $U$-complete numbering, as it can be found in Mal'cev's and Eršov's work. Originally, the following definition was stated in [12] and [4] for arbitrary collections of objects, rather than restricted to partial recursive functions.

Definition 2 ([12,4]) Let $U \subseteq \mathcal{P}$. A numbering $\psi$ is called $U$-complete numbering (or complete numbering for $U$ ) if $\mathcal{P}_{\psi}=U$ and there is an element $\perp \in U$ (called a "special element") with the following property: for every $f \in \mathcal{P}$, there is a $g \in \mathcal{R}$ such that for all $x$,

$$
\psi_{g(x)}= \begin{cases}\psi_{f(x)}, & \text { if } f(x) \downarrow \\ \perp, & \text { otherwise. }\end{cases}
$$

The use of $\perp$ for the special element suggests that $\perp$ is a least element. As the following theorem shows, this is indeed the case. The theorem seems to be folklore; the following proof is due to John Case.

Theorem 2 Consider any $U \subseteq \mathcal{P}$. If $\psi$ is $U$-complete numbering then the special element $\perp$ is the least element for $U$.

Proof. Suppose by way of contradiction that $\psi$ is $U$-complete numbering and the special element $\perp$ is not the least element of $U$. Then there is a $\psi_{i} \in U$ such that $\perp \nsubseteq \psi_{i}$. Thus, there exist $m, n$ such that $\perp(m) \downarrow=n$ and $\psi_{i}(m) \neq n$. It may be the case that $\psi_{i}(m) \uparrow$. Let $A$ be any recursively enumerable but not recursive set and, for all $x$, let

$$
f(x)= \begin{cases}i, & \text { if } x \in A ; \\ \uparrow, & \text { otherwise. }\end{cases}
$$

Clearly, $f$ is partial recursive. Since $\psi$ is $U$-complete, there exists a $g \in \mathcal{R}$ such that for all $x$,

$$
\psi_{g(x)}= \begin{cases}\psi_{f(x)}, & \text { if } f(x) \downarrow \\ \perp, & \text { otherwise. }\end{cases}
$$

That is,

$$
\psi_{g(x)}= \begin{cases}\psi_{i}, & \text { if } x \in A \\ \perp, & \text { if } x \notin A\end{cases}
$$


Thus, for all $x, x \in \bar{A}$ if and only if $\psi_{g(x)}(m) \downarrow=n$. This implies that $\bar{A}$ is recursively enumerable, a contradiction.

Mal'cev and Eršov prove the following facts about $U$-complete numberings.

\section{Theorem 3 ([12,4])}

(a) (Fixed Point Theorem, FPT) If $\psi$ is a U-complete numbering then, for every recursive function $f$, the equation $\psi_{f(n)}=\psi_{n}$ has a solution ("fixed point") n. Moreover, there is an algorithm which given a program for $f$ computes such a solution, i.e., there exists a recursive function $g$ such that, for all $n$, if $\varphi_{n}$ is recursive, the following holds:

$$
\psi_{g(n)}=\psi_{\varphi_{n}(g(n))} .
$$

(b) Every numbering which is equivalent to a $U$-complete numbering is isomorphic to the latter, and hence it is also $U$-complete.

(c) Every U-complete numbering has infinite padding.

(d) Every U-complete numbering satisfies Rice's Theorem, i.e., the only recursive index sets are the trivial ones.

Note that (b) does not imply that, for some fixed $U$, all $U$-complete numberings are equivalent. In fact, there exists a set $U$ of partial recursive functions that has $U$-complete numberings which are not all equivalent.

We now define the notion of $U$-acceptable numberings. As it will turn out, this notion is stronger than $U$-completeness. But using $U$-acceptability instead of $U$-completeness will simplify some of the proofs later on. Beside that, $U$-acceptable numberings have some interesting properties on their own.

Definition 3 Let $U \subseteq \mathcal{P}$ such that $U$ has a least element. A numbering $\psi \in$ $\mathcal{P}^{2}$ is called $U$-acceptable numbering (also called $U$-acceptable programming system, acceptable numbering for $U$ ) if the following properties are satisfied:

(a) $\mathcal{P}_{\psi}=U$

(b) for all numberings $\eta$, if $\mathcal{P}_{\eta} \subseteq U$, then $\eta \preceq \psi$, 
Since $\preceq$ is transitive and reflexive, all $U$-acceptable numberings are equivalent.

Note that the traditional notion of acceptable numbering is equivalent to $\mathcal{P}$-acceptable numbering. Parts (a) and (b) in the above definition are natural counterparts of traditional definition of acceptable numberings when we restrict ourselves to $U$ instead of $\mathcal{P}$. Further note, that $U$-acceptable numberings are defined only for $U$ having a least element. This is needed to get useful properties from $U$-acceptable numberings. A similar property was exploited by Mal'cev and Eršov in the definition of $U$-complete numberings; see the special element in Definition 2.

Proposition 1 If $U \subseteq \mathcal{P}$ is finite and has a least element, then there exists a $U$-acceptable numbering.

Proof. Assume $U=\left\{f_{0}, f_{1}, \ldots, f_{n}\right\}$ and $f_{0}=\perp$. Let $\psi$ be any acceptable numbering. For each $i \leq n$, let $\Delta_{i} \subseteq f_{i}$ be a finite function such that, for $j \leq n, \Delta_{i} \subseteq f_{j}$, if and only if $f_{i} \subseteq f_{j}$. Note that such $\Delta_{i}$ can easily be constructed.

The functions $\eta_{i}$ in $\eta$ are defined as follows. Let $m_{0}=0$. Go to stage 0 .

Stage $s$

1. For each $x \leq s$ such that $f_{m_{s}}(x) \downarrow$ in at most $s$ steps, let $\eta_{i}(x)=f_{m_{s}}(x)$.

2. If there are $j$ such that $f_{m_{s}} \subset f_{j}$, and $\Delta_{j} \subseteq \psi_{i}$ can be detected within $s$ steps, then let $m_{s+1}=$ least such $j$.

Otherwise, let $m_{s+1}=m_{s}$.

3. Go to stage $s+1$

End stage $s$

Fix $i$ and consider the computation of $\eta_{i}$. Note that making $f_{m_{s}} \subseteq \eta_{i}$ in step 1 above is consistent, since $f_{m_{s}} \subseteq f_{m_{s+1}}$ by definition of $m_{s+1}$, and thus, $f_{m_{0}} \subseteq f_{m_{1}} \subseteq f_{m_{2}} \cdots$. Therefore $\eta$ is a computable numbering.

Also, $\lim _{s \rightarrow \infty} m_{s} \downarrow=m$ such that $f_{m} \subseteq \psi_{i}$, and for all $m^{\prime} \leq n, \neg\left[f_{m} \subset\right.$ $\left.f_{m^{\prime}} \subseteq \psi_{i}\right]$. Hence, $\eta_{i}=f_{m}$, for some $m$ such that $f_{m} \subseteq \psi_{i}$ and for all $m^{\prime} \leq n$, $\neg\left[f_{m} \subset f_{m^{\prime}} \subseteq \psi_{i}\right]$.

It follows that $\eta_{i} \in U$, and also $U \subseteq \mathcal{P}_{\eta}$ is easy to see. Furthermore, if $\psi_{i} \in U$ then $\eta_{i}=\psi_{i}$. Let $\zeta$ be any subnumbering of $U$. Then $\zeta \preceq \psi$, since $\psi$ is acceptable. This, together with the previous remark yields $\zeta \preceq \eta$, and hence $\eta$ is $U$-acceptable. The proposition follows. 


\section{$3 \quad U$-Completeness and $U$-Acceptability}

We first show that every $U$-acceptable numbering is a $U$-complete numbering. Therefore, if we later on show the existence of a $U$-acceptable numbering with a certain property, we have also found a $U$-complete numbering with that property.

Theorem 4 If $\psi$ is $U$-acceptable then $\psi$ is $U$-complete.

Proof. Suppose $U \subseteq \mathcal{P}$ and $\psi$ is $U$-acceptable. Choose the least element of $U$ to be the special element $\perp$ in the definition of $U$-complete numbering. Let $f$ be any partial recursive function. Define $\eta$ as follows.

$$
\eta_{x}= \begin{cases}\psi_{f(x)}, & \text { if } f(x) \downarrow \\ \perp, & \text { if } f(x) \uparrow\end{cases}
$$

Note that first and second clauses of above definition are consistent, since $\perp$ is the least element of $\mathcal{P}_{\psi}$.

Clearly, $\eta$ is a subnumbering of $\psi$. Let $r \in \mathcal{R}$ be such that $\eta \preceq_{r} \psi$ (such $r$ exists since $\psi$ is $U$-acceptable).

Now, for all $x$, we have

$$
\psi_{r(x)}=\eta_{x}= \begin{cases}\psi_{f(x)}, & \text { if } f(x) \downarrow \\ \perp, & \text { otherwise }\end{cases}
$$

It follows that $\psi$ is a $U$-complete numbering.

The next two theorems show that $U$-acceptable numberings are a proper restriction of $U$-complete numberings.

The proof for the following theorem can be found in [4] for the general case, i.e., for enumerations of arbitrary objects. Here we give an easier version, specialized for partial recursive functions.

Theorem 5 Suppose $U \subseteq \mathcal{P}$ is recursively enumerable and there exists a least element $\perp$ of $U$. Then there exists a complete numbering $\psi$ for $U$.

Proof. Suppose $\eta$ is a numbering of $U$. Define $\psi$ as follows:

$\psi_{2 i}=\eta_{i}$.

$\psi_{2\langle i, j\rangle+1}$ is defined as follows. 
$\psi_{2\langle i, j\rangle+1}(x)$

In parallel execute steps 1 and 2.

1. For $x$ such that $\perp(x) \downarrow$, let $\psi_{2\langle i, j\rangle+1}(x)=\perp(x)$.

2. Let $i^{\prime}=i$ and $j^{\prime}=j$.

Loop

2.1 Wait until $\varphi_{i^{\prime}}\left(j^{\prime}\right) \downarrow$.

2.2 Suppose $\varphi_{i^{\prime}}\left(j^{\prime}\right)=k$.

2.3 If $k$ is even, then $\psi_{2\langle i, j\rangle+1}=\psi_{k}=\eta_{k / 2}$.

2.4 If $k$ is odd, then let $i^{\prime}, j^{\prime}$ (for next iteration) be such that $k=2\left\langle i^{\prime}, j^{\prime}\right\rangle+1$, and go to next iteration of the loop.

End Loop

End

Note that step 2.4 is essentially a simulation of $\psi_{k}$. It immediately follows that

$$
\psi_{2\langle i, j\rangle+1}= \begin{cases}\psi_{k}, & \text { if } \varphi_{i}(j) \downarrow=k ; \\ \perp, & \text { if } \varphi_{i}(j) \uparrow .\end{cases}
$$

Thus for every partial recursive function $\varphi_{i}, g(x)=2\langle i, x\rangle+1$, witnesses the requirement for complete numbering. Thus $\psi$ is a $U$-complete numbering with $\perp$ as the special element.

On the other hand there are infinite enumerable classes $U$ with a least element that do not have $U$-acceptable numberings.

Theorem 6 There exists an infinite recursively enumerable class $U$ with least element such that $U$ has no $U$-acceptable numbering.

Proof. Let $U=\{f \mid f$ has finite domain $\}$. Clearly $U$ is recursively enumerable and has $\emptyset$ as the least element. Suppose by way of contradiction that some $\psi$ is $U$-acceptable. Construct $\eta$ as follows.

$$
\eta_{i}(x)= \begin{cases}0, & \text { if } \varphi_{i}(i) \downarrow=j \text { and }\left[x=1 \text { or } \psi_{j}(x-1) \downarrow\right] ; \\ \uparrow, & \text { otherwise. }\end{cases}
$$

Clearly $\eta$ is a numbering. Since every function in $\mathcal{P}_{\psi}$ has finite domain, it follows that every $\eta_{i}$ has finite domain: if $\varphi_{i}(i) \uparrow$, then domain $\left(\eta_{i}\right)=\emptyset$; otherwise $\max \left(\operatorname{domain}\left(\eta_{i}\right)\right)=1+\max \left(\operatorname{domain}\left(\psi_{\varphi_{i}(i)}\right)\right)$. Now suppose $\varphi_{k} \in \mathcal{R}$ 
reduces $\eta$ to $\psi$. Then $\max \left(\operatorname{domain}\left(\eta_{k}\right)\right)=1+\max \left(\operatorname{domain}\left(\psi_{\varphi_{k}(k)}\right)\right)$. Thus $\eta_{k} \neq \psi_{\varphi_{k}(k)}$, a contradiction. Therefore $\psi$ is not $U$-acceptable.

Corollary 1 There exists $U \subseteq \mathcal{P}$ such that $U$ has $U$-complete numberings but no $U$-acceptable numberings.

Proof. Immediate by Theorems 5 and 6 .

\section{Results}

Let us first prove some general properties for complete numberings.

Theorem 7 (Fixed Point Theorem with Parameters) Let $U \subseteq \mathcal{P}$ and $\psi$ be $U$-complete. For all $h \in \mathcal{R}^{2}$ there exists $g \in \mathcal{R}$ such that, for all $y$,

$$
\psi_{g(y)}=\psi_{h(g(y), y)} .
$$

Proof. Define a function $u$ by $u(\langle x, y\rangle)=\varphi_{x}(\langle x, y\rangle)$. So there exists a recursive $r$ such that

$$
\psi_{r(\langle x, y\rangle)}= \begin{cases}\psi_{u(\langle x, y\rangle),} & \text { if, } u(\langle x, y\rangle) \downarrow \\ \perp, & \text { otherwise }\end{cases}
$$

Now consider any $h \in \mathcal{R}^{2}$. Let $n$ such that, for all $x, y, \varphi_{n}(\langle x, y\rangle)=$ $h(r(\langle x, y\rangle), y)$. Then $\varphi_{n}$ is clearly recursive and thus, for all $y, \varphi_{n}(\langle n, y\rangle) \downarrow$. Now, for all $y$,

$$
\psi_{r(\langle n, y\rangle)}=\psi_{u(\langle n, y\rangle)}=\psi_{\varphi_{n}(\langle n, y\rangle)}=\psi_{h(r(\langle n, y\rangle), y)} .
$$

Thus taking $g(y)=r(\langle n, y\rangle)$ satisfies the theorem.

The next theorem shows that a stronger formulation of Fixed Point Theorem also holds.

Theorem 8 (Double FPT) Let $U \subseteq \mathcal{P}$ and $\psi$ be a $U$-complete numbering. For all $f, g \in \mathcal{R}$ there exist $a, b$ such that

$$
\psi_{a}=\psi_{f(\langle a, b\rangle)} \text { and } \quad \psi_{b}=\psi_{g(\langle a, b\rangle)}
$$


Proof. Let $h(x, y)=f(\langle x, y\rangle)$. Then by parametric FPT (Theorem 7) there exists a recursive $r$ such that, for all $x, \psi_{r(x)}=\psi_{h(r(x), x)}=\psi_{f(\langle r(x), x\rangle)}$. Let $r^{\prime}(x)=g(\langle r(x), x\rangle)$. Now by FPT (Theorem 3(a)) there exists an $e$ such that $\psi_{e}=\psi_{r^{\prime}(e)}=\psi_{g(\langle r(e), e\rangle)}$.

Taking $a=r(e)$ and $b=e$ thus satisfies the theorem.

The proof of the following theorem can be found in [4]. (Actually, Eršov proves the theorem for the weaker notion of pre-complete numberings.)

Theorem 9 Suppose $U \subseteq \mathcal{P}$ and $\psi$ is a $U$-complete numbering.

(a) (Extended Rice's Theorem) Suppose $V \subseteq U$ is such that, for some $f, g \in U, f \subset g, f \in V$, and $g \notin V$. Then $I_{V}^{\psi}$ is not recursively enumerable.

(b) (Rice's Theorem) For all $V$ such that $\emptyset \subset V \subset U, I_{V}^{\psi}$ is not recursive.

For universal numberings, s-m-n-Theorem and composition were necessary to obtain acceptability; cf. Theorem 1 . This no longer holds for complete numberings, as the corollary to the next theorem shows; the theorem actually gives a stronger result.

\section{Theorem 10}

(a) There exists $U \subseteq \mathcal{P}$ such that there exist $U$-acceptable numberings, but no numbering of $U$ satisfies (even non-effective) s-m-n-Theorem.

(b) There exists $U \subseteq \mathcal{P}$ such that there exist $U$-acceptable numberings, but no numbering of $U$ has (even non-effective) composition.

Proof. (a) Choose $U=\{\emptyset, i d\}$, where $i d$ is the identity function. Clearly, $U$ has $U$-acceptable numberings by Proposition 1 . Let $\psi$ be any numbering for $U$. Since $\langle\cdot, \cdot\rangle$ is one-to-one and onto, there is a $k$ satisfying $k \neq\langle k, k\rangle$. Suppose by way of contradiction that $\psi$ satisfies s-m-n-Theorem and let $s$ be the s-m-n-function. (Note that we may even take $s$ to be non-computable for the following). Let $i$ be such that $\psi_{i}=i d$. Now $\psi_{s(i, k)}(k)=\psi_{i}(\langle k, k\rangle)=$ $i d(\langle k, k\rangle)=\langle k, k\rangle$. But since $k \neq\langle k, k\rangle$, this implies that $\psi_{s(i, k)} \notin\{\emptyset, i d\}$. A contradiction to the fact that $\psi$ is a numbering for $U$.

(b) Let $f, g$ be defined as follows. $f(x)=x+1$, and $g(x)=x^{2}$. Let $U=\{\emptyset, f, g\}$. Again, by Proposition 1, $U$ has $U$-acceptable numberings. However, $\lambda x . f(g(x)) \notin U$, and thus no composition exists. 


\section{Corollary 2}

(a) There exists $U \subseteq \mathcal{P}$ such that there exist $U$-complete numberings, but no numbering of $U$ satisfies (even non-effective) $s$-m-n-Theorem.

(b) There exists $U \subseteq \mathcal{P}$ such that there exist $U$-complete numberings, but no numbering of $U$ has (even non-effective) composition.

Proof. Immediate by Theorems 4 and 10.

On the other hand, it is easy to see that none of composition, s-m-nTheorem or KRT, nor any combination of the three, is sufficient for a numbering $\psi$ to be complete, even if $\mathcal{P}_{\psi}$ contains a least element. To see this, define $\psi$ as follows.

$$
\psi_{i}(x)= \begin{cases}j, & \text { if } i=2 j \\ \uparrow, & \text { if } i=2 j+1 .\end{cases}
$$

Obviously, the set $\mathcal{P}_{\psi}$ has a least element, $\psi$ has composition, satisfies the s-m-n-Theorem and KRT, but is not $\mathcal{P}_{\psi^{-}}$-complete. To see this, note for example that $f(x)=0$ has exactly one index in $\psi$ and therefore Rice's Theorem can not be satisfied by $\psi$, in contrast to what Theorem $3(\mathrm{~d})$ requires of complete numberings.

As the proofs of Theorem 10 and Corollary 2 show, the results hold for information-theoretic reasons: sometimes there is no function in $U$ that could realize the input-output behavior required by the programs that the s-m-n-Theorem or composition would generate. However, it is possible to strengthen Corollary 2 in order to show that it holds for computational reasons as well, i.e., there are $U$-complete numberings that satisfy non-effective s-m-n-Theorem and have non-effective composition, but no effective counterparts.

\section{Theorem 11}

(a) There exists $U \subseteq \mathcal{P}$ and a $U$-complete numbering $\psi$, such that $\psi$ satisfies non-effective $s$-m-n-Theorem, but no effective $s$-m-n-Theorem.

(b) There exists $U \subseteq \mathcal{P}$ and a $U$-complete numbering $\psi$, such that $\psi$ has non-effective composition, but no effective composition. 
Proof. Recall that $\varphi$ is an acceptable numbering (for $\mathcal{P}$ ). Without loss of generality, assume $\varphi_{0}=\emptyset$ for ease of notation; so $\varphi_{0}$ cannot witness effective s-m-n-Theorem or composition. We will describe two numberings $\psi$ below, one to prove part (a) and one to prove part (b). Let $U=\left\{\psi_{i} \mid i \in \mathbb{N}\right\}$ in both cases.

First we define $\psi_{\langle 0, j, x\rangle}$ and will use this "first half" of $\psi$ for both parts of the proof to follow. We will make sure that

$$
\psi_{\langle 0, j, x\rangle}= \begin{cases}\psi_{\varphi_{j}(x)}, & \text { if } \varphi_{j}(x) \downarrow \\ \emptyset, & \text { otherwise }\end{cases}
$$

Note that this will imply $\psi$ being $U$-complete (since for any partial recursive function $f=\varphi_{j}$, one can take $\left.g(x)=\langle 0, j, x\rangle\right)$. So define $\psi_{\langle 0, j, x\rangle}$ as follows:

Definition of $\psi_{\langle 0, j, x\rangle}$.

1. Let $j^{\prime}=j$ and $x^{\prime}=x$.

2. Loop

2.1 If $\varphi_{j^{\prime}}\left(x^{\prime}\right) \uparrow$, then $\psi_{\langle 0, j, x\rangle}$ is empty function.

2.2 If $\varphi_{j^{\prime}}\left(x^{\prime}\right) \downarrow=\left\langle i^{\prime \prime}, j^{\prime \prime}, x^{\prime \prime}\right\rangle$ with $i^{\prime \prime}>0$, then let $\psi_{\langle 0, j, x\rangle}=\psi_{\left\langle i^{\prime \prime}, j^{\prime \prime}, x^{\prime \prime}\right\rangle}$.

2.3 If $\varphi_{j^{\prime}}\left(x^{\prime}\right) \downarrow=\left\langle 0, j^{\prime \prime}, x^{\prime \prime}\right\rangle$, then let $j^{\prime}=j^{\prime \prime}$ and $x^{\prime}=x^{\prime \prime}$ and go to next iteration of the loop.

\section{End Loop}

End

Note that if the loop above is infinite, then $\psi_{\langle 0, j, x\rangle}$ is the empty function. Step 2.3 is essentially a simulation of $\psi_{\left\langle 0, j^{\prime \prime}, x^{\prime \prime}\right\rangle}$. Thus it immediately follows that

$$
\psi_{\langle 0, j, x\rangle}= \begin{cases}\psi_{\varphi_{j}(x)}, & \text { if } \varphi_{j}(x) \downarrow ; \\ \emptyset, & \text { otherwise. }\end{cases}
$$

Thus, whatever we do with $\psi_{\langle i, j, k\rangle}$, for $i \geq 1$, we have already ensured that $\psi$ is a $U$-complete numbering.

Also, note that for each $j, k$, either $\psi_{\langle 0, j, k\rangle}$ is the empty set, or $\psi_{\langle 0, j, k\rangle}=$ $\psi_{\left\langle i, j^{\prime}, k^{\prime}\right\rangle}$ for some $i>0$ and $j^{\prime}, k^{\prime} \in \mathbb{N}$.

(a) Let $r$ be such that $\varphi_{r}(i) \downarrow$ if and only if $\varphi_{i}(\langle i, 0,0\rangle, 0) \downarrow=\langle i, 0,0\rangle$ or $\varphi_{i}(\langle i, 0,0\rangle, 0) \downarrow=\left\langle 0, j^{\prime}, k^{\prime}\right\rangle$, and $\psi_{\left\langle 0, j^{\prime}, k^{\prime}\right\rangle}$, eventually follows $\psi_{\langle i, 0,0\rangle}$ in the definition of $\psi_{\left\langle 0, j^{\prime}, k^{\prime}\right\rangle}$ above. Note that there exists such a $\varphi_{r}$.

We now go on to define $\psi_{\langle i, j, k\rangle}$ for $i \geq 1$. Here we will try to let $\psi$ satisfy non-effective s-m-n-Theorem but not the effective s-m-n-Theorem. 
Recall that $F_{n}, n \geq 0$, is a canonical enumeration of all and only the finite functions. For $i>0$, and $j+k>0$, let

$$
\psi_{\langle i, j, k\rangle}(x)= \begin{cases}i, & \text { if } x \in \operatorname{domain}\left(F_{j+k-1}\right) ; \\ \uparrow, & \text { otherwise. }\end{cases}
$$

Note that every finite function with range $=\{i\}$ is represented in the construction above.

We now define $\psi_{\langle i, 0,0\rangle}$ for $i>0$. Let

$$
\psi_{\langle i, 0,0\rangle}(\langle x, y\rangle)= \begin{cases}i, & \Phi_{r}(i)>\langle x, y\rangle \text { or } \\ & {\left[x=0 \wedge y=\min \left(\left\{y^{\prime} \mid\left\langle 0, y^{\prime}\right\rangle>y^{\prime}>\Phi_{r}(i)\right\}\right)\right.} \\ \uparrow, & \text { otherwise. }\end{cases}
$$

Note that for any constant $c$, if domain of $\psi_{i}$ is finite, then domain of $\lambda x . \psi_{i}(\langle c, x\rangle)$ is also finite. Using the above, it is easy to verify that $\psi$ satisfies non-effective s-m-n-Theorem.

We now show that $\psi$ does not satisfy effective s-m-n-Theorem. Suppose by way of contradiction, that $\varphi_{i}$ witnesses the effective s-m-n-Theorem for $\psi$. By assumption on $\varphi$ we have $i>0$. Now, consider the following cases:

Case 1: $\varphi_{r}(i) \downarrow$.

In this case, $\psi_{\varphi_{i}(\langle i, 0,0\rangle, 0)}=\psi_{\langle i, 0,0\rangle}$. However, $\psi_{\langle i, 0,0\rangle}(\langle 0, y\rangle) \neq \psi_{\langle i, 0,0\rangle}(y)$, where $y=\min \left(\left\{y^{\prime} \mid\left\langle 0, y^{\prime}\right\rangle>y^{\prime}>\Phi_{r}(i)\right\}\right)$.

Case 2: $\varphi_{r}(i) \uparrow$.

In this case $\psi_{\langle i, 0,0\rangle}$ is the constant $i$ function, and for any $\left\langle i^{\prime}, j^{\prime}, k^{\prime}\right\rangle$, $\psi_{\left\langle i^{\prime}, j^{\prime}, k^{\prime}\right\rangle}=\psi_{\langle i, 0,0\rangle}$ if and only if $\left\langle i^{\prime}, j^{\prime}, k^{\prime}\right\rangle=\langle i, 0,0\rangle$, or $i^{\prime}=0$, and $\psi_{\left\langle i^{\prime}, j^{\prime}, k^{\prime}\right\rangle}$ eventually follows $\psi_{\langle i, 0,0\rangle}$ in the construction above. Therefore $\psi_{\varphi_{i}(\langle i, 0,0\rangle, 0)} \neq$ $\psi_{\langle i, 0,0\rangle}$ holds. Thus, $\varphi_{i}$ does not witness effective s-m-n-Theorem for $\psi$.

The above case distinction proves part (a).

(b) Let $r$ be such that $\varphi_{r}(i) \downarrow$ if and only if $\varphi_{i}(\langle i, 0,0\rangle,\langle i, 0,0\rangle) \downarrow=\langle i, 0,0\rangle$ or $\varphi_{i}(\langle i, 0,0\rangle,\langle i, 0,0\rangle) \downarrow=\left\langle 0, j^{\prime}, k^{\prime}\right\rangle$, and $\psi_{\left\langle 0, j^{\prime}, k^{\prime}\right\rangle}$, eventually follows $\psi_{\langle i, 0,0\rangle}$ in the definition of $\psi_{\left\langle 0, j^{\prime}, k^{\prime}\right\rangle}$ above. Note that there exists such a $\varphi_{r}$.

We now again define $\psi_{\langle i, j, k\rangle}$ for $i \geq 1$. For $i>0$, if $j \neq 0$, or $k>1$, then let $\psi_{\langle i, j, k\rangle}=\emptyset$.

Let $p_{i}$ denote the $i$-th prime number. For $i>0$, let

$$
\psi_{\langle i, 0,0\rangle}(x)= \begin{cases}p_{i}, & \text { if } x=p_{i} ; \\ p_{i}^{2}, & \text { if } \varphi_{r}(i) \downarrow \text { and } x=p_{i}^{3} ; \\ p_{i}^{3}, & \text { if } \varphi_{r}(i) \downarrow \text { and } x=p_{i}^{2} ; \\ \uparrow, & \text { otherwise. }\end{cases}
$$




$$
\psi_{\langle i, 0,1\rangle}(x)= \begin{cases}p_{i}, & \text { if } \varphi_{r}(i) \downarrow \text { and } x=p_{i} ; \\ p_{i}^{2}, & \text { if } \varphi_{r}(i) \downarrow \text { and } x=p_{i}^{2} \\ p_{i}^{3}, & \text { if } \varphi_{r}(i) \downarrow \text { and } x=p_{i}^{3} ; \\ \uparrow, & \text { otherwise. }\end{cases}
$$

By the use of the prime numbers $p_{i}$ it is easy to see that, (a) for all functions $f$ in $U$, domain and range of $f$ are same, and (b) for all functions $f$ and $g$ in $U$, either the domains of $f$ and $g$ are equal, or they are disjoint. Using this property, it is easy to verify that non-effective composition holds for $\psi$.

Suppose by way of contradiction, that $\varphi_{i}$ witnesses effective composition for $\psi$. By assumption on $\varphi$ we have $i>0$.

We consider two cases.

Case 1: $\varphi_{r}(i) \downarrow$.

In this case, $\psi_{\varphi_{i}(\langle i, 0,0\rangle,\langle i, 0,0\rangle)}=\psi_{\langle i, 0,0\rangle}$. However, this does not witness effective composition, since $\psi_{\langle i, 0,0\rangle} \circ \psi_{\langle i, 0,0\rangle} \neq \psi_{\langle i, 0,0\rangle}$.

Case 2: $\varphi_{r}(i) \uparrow$.

In this case, $\psi_{\langle i, 0,0\rangle} \circ \psi_{\langle i, 0,0\rangle}=\psi_{\langle i, 0,0\rangle}$. Moreover, if $\psi_{\left\langle i^{\prime}, j^{\prime}, k^{\prime}\right\rangle}=\psi_{\langle i, 0,0\rangle}$, then $\left\langle i^{\prime}, j^{\prime}, k^{\prime}\right\rangle=\langle i, 0,0\rangle$ or $i^{\prime}=0$, and $\psi_{\left\langle i^{\prime}, j^{\prime}, k^{\prime}\right\rangle}$ eventually follows $\psi_{\langle i, 0,0\rangle}$ in the above algorithm. Thus, $\varphi_{i}$ cannot witness effective composition for $\psi$.

It follows from the above cases that $\psi$ does not have effective composition.

For the stronger notion of $U$-acceptability we get a nice contrast: for $U$ complete numberings, there is a difference between effective and non-effective s-m-n-Theorem, resp. composition. With $U$-acceptability, it is not possible to strengthen Theorem 10 to hold for computational reasons.

Theorem 12 Let $U \subseteq \mathcal{P}$ and $\psi$ be $U$-acceptable.

(a) $\psi$ satisfies non-effective $s$-m-n-Theorem if and only if $\psi$ satisfies effective $s$-m-n-Theorem.

(b) $\psi$ has non-effective composition if and only if $\psi$ has effective composition.

Proof. We prove part (a); the proof for (b) is similar. Assume $U$ and $\psi$ as in the hypotheses of the theorem. Let $s^{\prime}$ be a non-effective s-m-n-function for $\psi$, i.e., for all $x, y, z$,

$$
\psi_{s^{\prime}(x, y)}(z)=\psi_{x}(\langle y, z\rangle) .
$$


Note that this implies $\psi_{s^{\prime}(x, y)} \in U$. Define a numbering $\eta$, for all $x, y, z$, as follows:

$$
\eta_{\langle x, y\rangle}(z)=\psi_{x}(\langle y, z\rangle)
$$

Obviously, $\eta$ is a subnumbering of $\psi$ and therefore $\eta \preceq_{r} \psi$ for some recursive $r$. Define a recursive function $s$ by $s(x, y)=r(\langle x, y\rangle)$ for all $x, y$. This yields

$$
\psi_{s(x, y)}=\psi_{r(\langle x, y\rangle)}=\eta_{\langle x, y\rangle}=\psi_{s^{\prime}(x, y)}
$$

and thus $s$ witnesses effective s-m-n-Theorem.

The following theorem points out that Theorem 10 illustrates a general fact. Namely that the property of satisfying the s-m-n-Theorem - or composition, respectively - does not depend on the choice of a particular $U$ acceptable numbering, but on the choice of $U$ (and the choice of the used pairing function, as Theorem 15 will show).

Theorem 13 Let $U \subseteq \mathcal{P}$.

(a) If there exists a $U$-acceptable numbering satisfying the $s$-m-n-Theorem then every $U$-acceptable numbering satisfies the s-m-n-Theorem.

(b) If there exists a $U$-acceptable numbering with composition, then every $U$-acceptable numbering has composition.

Proof. We only show part (a); part (b) is similar. Suppose $\psi, \eta$ are $U$ acceptable numberings, where $\psi$ satisfies s-m-n-Theorem. Let $r$ and $r^{\prime}$ be recursive functions such that $\psi \preceq_{r} \eta$, and $\eta \preceq_{r^{\prime}} \psi$. Such functions exist by definition of $U$-acceptable numbering. Let $s^{\prime}$ be the s-m-n-function of $\psi$. Define $s(x, y)=r\left(s^{\prime}\left(r^{\prime}(x), y\right)\right)$. Now $\eta_{s(x, y)}(z)=\eta_{r\left(s^{\prime}\left(r^{\prime}(x), y\right)\right)}(z)=$ $\psi_{s^{\prime}\left(r^{\prime}(x), y\right)}(z)=\psi_{r^{\prime}(x)}(\langle y, z\rangle)=\eta_{x}(\langle y, z\rangle)$ and $s$ is the desired s-m-n-function for $\eta$.

The situation is more complicated for complete numberings, as witnessed by the following theorem.

Theorem 14 There exists a $U \subseteq \mathcal{P}$ such that

(a) there exists a U-complete numbering which satisfies effective $s$-m-nTheorem. 
(b) there exists a U-complete numbering which does not satisfy effective $s-m$ - $n$-Theorem.

Proof. For proofs of both parts let $U=\{f \mid f$ has finite domain $\}$. Recall that $F_{0}, F_{1}, \ldots$ is a recursive enumeration of $U$ such that for each $i$, one can effectively (in $i$ ) determine card $\left(\operatorname{domain}\left(F_{i}\right)\right.$ ).

(a) We give a numbering $\eta$ which is $U$-complete and satisfies effective s-m-n theorem.

Let $\eta_{3 i}=F_{i}$.

$\eta_{3 i+1}$ and $\eta_{3 i+2}$, for each $i$, will be defined below. We will make sure that

(i) $\eta_{3 i+1}, \eta_{3 i+2}$ have finite domain,

(ii) $\eta_{3\langle i, j\rangle+1}=\lambda x \cdot \eta_{i}(\langle j, x\rangle)$, and

(iii)

$$
\eta_{3\langle i, j\rangle+2}= \begin{cases}\eta_{\varphi_{i}(j)}, & \text { if } \varphi_{i}(j) \downarrow ; \\ \emptyset, & \text { otherwise. }\end{cases}
$$

Thus, $s(i, j)=3\langle i, j\rangle+1$ would witness the satisfaction of effective s-mn-Theorem. Also $\eta$ would be $U$-complete, since for any $\varphi_{i}, g(j)=3\langle i, j\rangle+2$ witnesses the requirement for $U$-complete numbering.

We now continue with the definition of $\eta_{3 i+1}$ and $\eta_{3 i+2}$, for each $i$.

$\eta_{3\langle i, j\rangle+k}$, for $i, j \in \mathbb{N}, k \in\{1,2\}$

1. Let $i^{\prime}=i, j^{\prime}=j, k^{\prime}=k$. Let $\ell=0$.

(* We will be using numbers $n_{i}$ later on. Inductively, $n_{i}$ has been defined for $\left.1 \leq i \leq \ell^{*}\right)$.

Loop

2 If $k=1$, then

$2.1 \quad$ let $n_{\ell+1}=j^{\prime}$

2.2 let $\ell=\ell+1$;

2.3 Suppose $i^{\prime}=3\left\langle i^{\prime \prime}, j^{\prime \prime}\right\rangle+k^{\prime \prime}$, for some $i^{\prime \prime}, j^{\prime \prime} \in \mathbb{N}$ and $k^{\prime \prime} \in$ $\{0,1,2\}$.

$2.4 \quad$ If $k^{\prime \prime}=0$, then let $\eta_{3\langle i, j\rangle+k}(x)=\eta_{i^{\prime}}\left(\left\langle n_{1},\left\langle n_{2}, \ldots,\left\langle n_{\ell}, x\right\rangle\right\rangle\right\rangle\right)$.

2.5 Else let $i^{\prime}=i^{\prime \prime}, j^{\prime}=j^{\prime \prime}$ and $k^{\prime}=k^{\prime \prime}$, and go to the next iteration of the loop.

3 If $k=2$, then

3.1 Wait until $\varphi_{i^{\prime}}\left(j^{\prime}\right)$ converges (if ever).

3.2 Suppose $\varphi_{i^{\prime}}\left(j^{\prime}\right)=3\left\langle i^{\prime \prime}, j^{\prime \prime}\right\rangle+k^{\prime \prime}$, for some $i^{\prime \prime}, j^{\prime \prime} \in \mathbb{N}$ and $k^{\prime \prime} \in\{0,1,2\}$. 
3.3 If $k^{\prime \prime}=0$ and $\ell=0$, then let $\eta_{3\langle i, j\rangle+k}(x)=\eta_{w}(x)$, where $w=\varphi_{i^{\prime}}\left(j^{\prime}\right)$.

$3.4 \quad$ Else If $k^{\prime \prime}=0$ and $\ell>0$, then let $\eta_{3\langle i, j\rangle+k}(x)=\eta_{w}\left(\left\langle n_{1},\left\langle n_{2}, \ldots,\left\langle n_{\ell}, x\right\rangle\right\rangle\right\rangle\right)$, where $w=\varphi_{i^{\prime}}\left(j^{\prime}\right)$.

3.5 Else let $i^{\prime}=i^{\prime \prime}, j^{\prime}=j^{\prime \prime}$ and $k^{\prime}=k^{\prime \prime}$, and go to the next iteration of the loop.

End Loop

End

Intuitively, one may consider step 2 of the loop as simulation of $\eta_{i^{\prime}}$ (with $\left\langle n_{\ell}, x\right\rangle$ replacing $x$ for input) and step 3 as simulation of $\eta_{\varphi_{i^{\prime}}\left(j^{\prime}\right)}$. That is, $\eta_{3\langle i, j\rangle+1}(x)=\eta_{i}(\langle j, x\rangle), \eta_{3\langle i, j\rangle+2}(x)=\eta_{\varphi_{i}(j)}(x)$ (where right hand side becomes $\emptyset$, if $\left.\varphi_{i}(j) \uparrow\right)$.

Using above, it is easy to verify that the construction satisfies properties (ii) and (iii). Property (i) trivially follows, since $\eta_{w}$ is finite for each $w$ divisible by 3 (see steps 2.4, 3.3 and 3.4).

(b) We give a numbering $\psi$ which is $U$-complete, but does not satisfy effective s-m-n-Theorem. The idea is to use $\psi_{3\langle i, j\rangle}$, and $\psi_{3\langle i, j\rangle+1}$ to ensure that $\psi$ is a $U$-complete numbering, and to use $\psi_{3\langle i, j\rangle+2}$ to spoil any potential candidate for effective s-m-n-Theorem.

Let $\eta_{3 i}=F_{i}$.

$\eta_{3\langle i, j\rangle+1}$ is defined using the following procedure.

$\eta_{3\langle i, j\rangle+1}$, for $i, j \in \mathbb{N}$.

1. Let $i^{\prime}=i, j^{\prime}=j$.

Loop

2.1 Wait until $\varphi_{i^{\prime}}\left(j^{\prime}\right)$ converges (if ever).

2.2 Suppose $\varphi_{i^{\prime}}\left(j^{\prime}\right)=3 w+k$, for some $w \in \mathbb{N}$ and $k \in\{0,1,2\}$.

2.3 If $k=0$ or $k=2$, then let $\eta_{3\langle i, j\rangle+1}(x)=\eta_{3 w+k}(x)$.

2.4 Else suppose $w=\left\langle i^{\prime \prime}, j^{\prime \prime}\right\rangle$. Go to the next iteration of the loop, with $i^{\prime}=i^{\prime \prime}$ and $j^{\prime}=j^{\prime \prime}$.

End Loop

End 
Note that, above construction makes

$$
\psi_{3\langle i, j\rangle+1}= \begin{cases}\psi_{\varphi_{i}(j)}, & \text { if } \varphi_{i}(j) \downarrow ; \\ \emptyset, & \text { otherwise }\end{cases}
$$

Thus, assuming each $\psi_{3 w+2} \in U$, above construction ensures that $\psi$ is $U$-complete numbering (since for any $\varphi_{i}$, one could take $g(j)=3\langle i, j\rangle+1$, and thus satisfy the requirement for $U$-complete numbering).

We now give description of $\psi_{3 w+2}$, for $w \in \mathbb{N}$. Without loss of generality assume that $\langle 0,0\rangle=0$ and $\langle 0,1\rangle \notin\{0,1\}$. We will make sure that each $R_{i}$ is satisfied, where

$R_{i}$ : either $\varphi_{i}(\langle 3 i+2,0\rangle) \uparrow$, or, $\psi_{\varphi_{i}(\langle 3 i+2,0\rangle)}(x) \neq \psi_{3 i+2}(\langle 0, x\rangle)$, for some $x \in\{0,1\}$.

Note that if each $R_{i}$ is satisfied, then we will have that $\psi$ does not satisfy effective s-m-n-Theorem.

We define $\psi_{3 i+2}$ as follows. For each $i, \psi_{3 i+2}(0)=0$, and $\psi_{3 i+2}(x) \uparrow$, for $x \notin\{0,\langle 0,1\rangle\}$. In particular, $\psi_{3 i+2}(1) \uparrow$.

We define $\psi_{3 i+2}(\langle 0,1\rangle)$ as follows.

Definition of $\psi_{3 i+2}(\langle 0,1\rangle)$ for $i \in \mathbb{N}$.

1. If $\varphi_{i}(\langle 3 i+2,0\rangle) \uparrow$, then $\psi_{3 i+2}(\langle 0,1\rangle)=\uparrow$.

2. Else,

Suppose $\varphi_{i}(\langle 3 i+2,0)\rangle=3 m+r$, where $r \in\{0,1,2\}$.

If $r \in\{0,2\}$, then go to step 3 with $w=m$ and $k=r$.

Else If $r=1$, and the procedure for $\psi_{3 m+1}$ eventually follows some $\psi_{3 w+k}$, where $k \in\{0,2\}$, then go to step 3 with this $w, k$.

Else $\psi_{3 i+2}(\langle 0,1\rangle)=\uparrow$.

3. If $k=0$ and $\psi_{3 w}(1) \downarrow$, then let $\psi_{3 i+2}(\langle 0,1\rangle)=\psi_{3 w}(1)+1$.

Else (in this case $k=2$ or $k=0$ and $\left.\psi_{3 w}(1) \uparrow\right)$, let $\psi_{3 i+2}(\langle 0,1\rangle)=1$.

(* note that one can determine effectively whether $\psi_{3 w}(1)=F_{w}(1) \uparrow *$ ). End

For each $i$, we claim that $R_{i}$ is satisfied. Suppose $\varphi_{i}(3 i+2,0) \downarrow=3 m+r$ (otherwise we are done).

We consider the following cases.

Case 1: $r=1$ and $\psi_{3 m+r}$ does not eventually follow some $\psi_{3 w+k}$, for $k \in\{0,2\}$. 
In this case $\psi_{3 m+r}=\emptyset$. Thus, we have that $\psi_{3 i+2}(\langle 0,0\rangle)=0 \neq \psi_{3 m+r}(0) \uparrow$. Case 2: $r \in\{0,2\}$ or $\psi_{3 m+r}$ eventually follows some $\psi_{3 w+k}$, where $k \in$ $\{0,2\}$.

In this case by step 3 of the construction, we have that $\psi_{3 i+2}(\langle 0,1\rangle) \neq$ $\psi_{3 m+r}(1)$.

From the above cases it follows that $\varphi_{i}$ cannot act as s-m-n function. Thus effective s-m-n-Theorem does not hold for $\psi$.

In Definition 1, the formulation of the s-m-n-Theorem used a pairing function $\langle\cdot, \cdot\rangle$. Unfortunately, the used pairing function might be the deciding factor whether $U$-complete numberings for a certain set $U$ satisfy the s-m-nTheorem or not.

Theorem 15 There exists $U \subseteq \mathcal{P}$ and pairing functions $\langle\cdot, \cdot \cdot,\langle\langle\cdot, \cdot\rangle\rangle$, such that $U$ has $U$-acceptable numberings satisfying $s-m-n$-Theorem when $\langle\cdot, \cdot\rangle$ is used, and $U$ has no $U$-complete numberings satisfying s-m-n-Theorem when using $\langle\langle\cdot, \cdot\rangle\rangle$.

Proof. Let $f$ be such that $f(x)=0$, if $x$ is odd and $f(x)=1$, if $x$ is even. Let $U=\{\emptyset, f\}$. Suppose $\langle\cdot, \cdot\rangle$ has the property that $\langle x, y\rangle$ is odd if and only if $y$ is odd. It is easy to define a pairing function with these constraints, just use a very simple "digit-merging" algorithm: it places the digits of the first argument on even digits in the result and the digits of the second arguments on the odd digits in the result, "sometimes" adding or removing zeros. (For example $\langle 0,0\rangle=0,\langle 0,1\rangle=1,\langle 1,0\rangle=10,\langle 400,1\rangle=400001,\langle 2,100\rangle=$ 10020 and so on.) Obviously, the algorithm has the desired properties. Then, clearly, any numbering for $U$ satisfies s-m-n-Theorem: $s(i, x)=i$ can be used. Suppose $\langle\langle\cdot, \cdot\rangle\rangle$ has the property that $\langle\langle x, y\rangle\rangle$ is odd if and only if $y$ is even. This can easily be obtained from the above pairing. Then no numbering for $U$ satisfies s-m-n-Theorem. This is so, since $\lambda y . f(\langle\langle x, y\rangle\rangle)$ does not belong to $U$.

Thus for some $U$, we may have s-m-n theorem in all acceptable/complete numberings (for example for $U=\{\emptyset$, Zero $\}$, where Zero is everywhere 0 function); for some $U$ we may have s-m-n theorem in no acceptable numbering under all pairing functions (Theorem 10 (a)); and for some $U$ we may or may not have s-m-n theorem in acceptable (complete) numberings based on the pairing function (Theorem 15).

The next result shows, that effective composition does not entail s-m-nTheorem for $U$-complete numberings. 
Theorem 16 There exists $U \subset \mathcal{P}$ such that

(a) there exists a $U$-acceptable numbering with effective composition,

(b) there exists no $U$-complete numbering satisfying (even non-effective) $s-m$-n-Theorem.

Proof. Let $U=\{\emptyset, i d\}$ and $\psi$ be any $U$-acceptable numbering. Such a numbering exists by Proposition 1. Now, $\psi_{i}(0) \downarrow$ if and only if $\psi_{i}=i d$. Define a numbering $\eta$ by

$$
\eta_{\langle x, y\rangle}= \begin{cases}i d, & \text { if } \psi_{x}(0) \downarrow \text { and } \psi_{y}(0) \downarrow ; \\ \emptyset, & \text { otherwise. }\end{cases}
$$

Since $\psi$ is $U$-acceptable and clearly $\mathcal{P}_{\eta} \subseteq \mathcal{P}_{\psi}$, there is an $r \in \mathcal{R}$ such that $\eta \preceq_{r} \psi$ and therefore

$$
\psi_{r(\langle x, y\rangle)}= \begin{cases}i d, & \text { if } \psi_{x}(0) \downarrow \text { and } \psi_{y}(0) \downarrow ; \\ \emptyset, & \text { otherwise }\end{cases}
$$

So defining $\operatorname{comp}(x, y)=r(\langle x, y\rangle)$ yields effective composition.

The fact, that the numbering $\psi$ can not satisfy s-m-n-Theorem is proved in Theorem 10(a).

The proof actually shows, that every $U$-acceptable numbering of $\{\emptyset, i d\}$ has effective composition. Note that it is not possible to drop " $U$-acceptable" here, since there exist numberings of $\{\emptyset, i d\}$, as can be proved using standard diagonalization techniques, that do not have effective composition.

On the other hand, s-m-n-Theorem does not imply composition in complete numberings and therefore the two concepts are independent in this context.

Theorem 17 There exists $U \subseteq \mathcal{P}$ such that

(a) there exists a $U$-acceptable numbering satisfying the effective $s$-m-nTheorem,

(b) there exists no numbering $\psi$, such that $\mathcal{P}_{\psi}=U$ and $\psi$ has (even noneffective) composition.

Corollary 3 There exists $U \subseteq \mathcal{P}$ such that $U$ has a $U$-complete numbering satisfying $s$-m-n-Theorem but no $U$-complete numbering has (even noneffective) composition. 
Proof. (of Theorem 17) Let $f(x)=0$ if $x$ is odd and $f(x)=1$ if $x$ is even and define a function $g$ by $g(x)=2$ if $x$ is even and $g(x)=0$ if $x$ is odd. Let $U=\{\emptyset, f, g\}$. Since $f(g(x))=1$ for all $x$, no numbering for $U$ can have composition. Let $\langle\cdot, \cdot\rangle$ be the pairing function defined in the proof of Theorem 15. By Proposition 1 there is a $U$-acceptable numbering $\psi$. Now it is easy to see, that taking $s(x, y)=x$ for all $x, y$ and using the properties of $\langle\cdot, \cdot\rangle$ yields effective s-m-n-Theorem for $\psi$.

We now turn our attention to KRT. Riccardi proves in [13] that effective KRT and FPT are independent in what he calls "programmable numberings". A sequence of functions $\left(\zeta_{i}\right)_{i \geq 0}$ is a programmable numbering if $\left\{\zeta_{i} \mid i \in \mathbb{N}\right\}=$ $\mathcal{P}$ and there exists a recursive $f$ such that $\zeta_{f(i)}=\varphi_{i}$ for all $i$. It is not required that the function $\lambda i, x . \zeta_{i}(x)$ is computable. Acceptable numberings of course satisfy both a KRT and a FPT. As Theorem 18 shows, this does not hold for $U$-acceptable numberings. Therefore, it does not hold for $U$-complete numberings as well.

Theorem 18 There exists $U \subseteq \mathcal{P}$ that has $U$-acceptable numberings but no numbering for $U$ satisfies $K R T$.

Proof. Let $U=\{\emptyset, i d\}$. By Proposition 1, there exist $U$-acceptable numberings. Suppose by way of contradiction that $\psi$ is a numbering for $U$ which satisfies KRT. Suppose $\psi_{i}=i d$ and, for all $x, \psi_{e}(x)=\psi_{i}(\langle e, x\rangle)=\langle e, x\rangle$. But then $\psi_{e} \notin U$.

As in Theorem 10, the argument above is information-theoretic.

Although for the $U$ exhibited in Theorem 18 no numbering satisfying KRT exists, an analogue to Theorem 13 does not hold for KRT in general in $U$-acceptable numberings, and therefore it also does not hold for $U$-complete numberings.

Theorem 19 There exists $U \subseteq \mathcal{P}$, such that

(a) there exists a $U$-acceptable numbering satisfying effective $K R T$.

(b) there exists a $U$-acceptable numbering not satisfying KRT (not even non-effective one).

ProOF. We assume that pairing function is monotonically increasing in both its arguments (in particular $\langle 0,0\rangle=0$ ) and $\langle i, j\rangle>j$, for $\langle i, j\rangle \neq\langle 0,0\rangle$. (This 
is more for ease of writing the proof, than a real necessity). Let code $(x)=x$. For $n \geq 2$, let code $\left(x_{1}, x_{2}, \ldots, x_{n}\right)=\left\langle x_{1}, \operatorname{code}\left(x_{2}, x_{3}, \ldots, x_{n}\right)\right\rangle$. Intuitively, code is a specific mechanism to extend the pairing function to multiple arguments. We will need the following property of code. Each $z \in \mathbb{N}$ can be uniquely expressed as $z=\operatorname{code}\left(e_{1}, e_{2}, \ldots, e_{n}, 0\right)$, where $e_{n}>0$ (here $n$ may be 0 , i.e. $z=\operatorname{code}(0))$. Intuitively, if $z=0$, then $n=0$. If $z \neq 0$, then $e_{1}=\pi_{1}(z)$, and $e_{2}, e_{3}, \ldots$, are determined inductively from $\pi_{2}(z)$. Note that we have assumed that the pairing function is increasing in both its arguments.

Define $f(x)=x, g_{\left(e_{1}\right)}(x)=f\left(\left\langle e_{1}, x\right\rangle\right)$ and further $g_{\left(e_{1}, \ldots, e_{n}, e_{n+1}\right)}(x)=$ $g_{\left(e_{1}, \ldots, e_{n}\right)}\left(\left\langle e_{n+1}, x\right\rangle\right)$.

Note that $g_{\left(e_{1}, \ldots, e_{n}\right)}(x)=f\left(\operatorname{code}\left(e_{1}, \ldots, e_{n}, x\right)\right)$.

Let $U=\{f, \emptyset\} \cup\left\{g_{\left(e_{1}, e_{2}, \ldots, e_{n}\right)} \mid n \geq 1 \wedge(\forall j \mid 1 \leq j \leq n)\left[e_{j}\right.\right.$ is odd $\left.]\right\}$.

Define $\psi$ as follows:

Let

$$
\begin{gathered}
\psi_{2 i}= \begin{cases}f, & \text { if } \varphi_{i}(0)=\operatorname{code}(0)=0 ; \\
g_{\left(e_{1}, \ldots, e_{n}\right)}, & \text { if } \varphi_{i}(0)=\operatorname{code}\left(e_{1}, \ldots, e_{n}, 0\right)>0, \text { and } \\
\emptyset, & e_{1}, \ldots, e_{n} \text { are all odd; } \\
\text { otherwise. }\end{cases} \\
\psi_{2 i+1}= \begin{cases}g_{(2 i+1)}, & \text { if } \psi_{i}(0)=\operatorname{code}(0)=0 ; \\
g_{\left(e_{1}, \ldots, e_{n}, 2 i+1\right)}, & \text { if } \psi_{i}(0)=\operatorname{code}\left(e_{1}, \ldots, e_{n}, 0\right)>0, \text { and } \\
\emptyset, & e_{1}, \ldots, e_{n} \text { are all odd } ; \\
\emptyset, & \text { otherwise. }\end{cases}
\end{gathered}
$$

Clearly, $\psi$ is $U$-acceptable numbering, and $\psi_{2 i+1}(x)=\psi_{i}(\langle 2 i+1, x\rangle)$. Thus $\psi$ satisfies effective KRT.

Now define $\eta$ as follows:

$\eta_{2 i}=\psi_{i}$, and $\eta_{2 i+1}=\emptyset$. Clearly, $\eta$ is $U$-acceptable too. However $\eta$ does not satisfy KRT, since for odd $e, \eta_{e}=\emptyset$, and for even $e, \lambda x . f(\langle e, x\rangle) \notin$ $U$.

Note that Theorem 12 does not hold if we use KRT instead of s-m-nTheorem or composition. Instead, we get an analogous result to Theorem 11 for both $U$-complete and $U$-acceptable numberings.

Theorem 20 There exists $U \subseteq \mathcal{P}$ and a $U$-acceptable numbering $\psi$ that satisfies non-effective KRT but not effective KRT. 
ProOf. Let $\eta^{0}, \eta^{1}, \ldots$ denote a recursive enumeration of all numberings. We will describe a numbering $\psi$ below. We will take $U=\left\{\psi_{i} \mid i \in \mathbb{N}\right\}$ and $\psi$ will satisfy the constraints of the theorem. The least element of $U$ will be $\emptyset$. Note, that since $\psi$ will be $U$-acceptable, there are infinitely many $\psi$-programs for $\emptyset$ : just recall that every $U$-acceptable numbering is $U$-complete by Theorem 4 and every $U$-complete numbering has infinite padding by Theorem 3(c). Thus, in the proof we need not worry about (non-effective) KRT being satisfied for finite domain functions in $U$. To see this, consider any finite function $f \in U$. Let $w$ be such that, for all $e \geq w$, for all $x, f(\langle e, x\rangle) \uparrow$. Let $e \geq w$, be such that $\psi_{e}=\emptyset$. Then clearly, for all $x, \psi_{e}(x)=f(\langle e, x\rangle)=\uparrow$.

$U$ constructed will have following property: for all $f, g \in U, f(0)=g(0)$ implies $f=g$ (so in essence $f(0)$ determines the element of $U$ ). This will make it easy to make $\psi$ acceptable, by defining $\psi_{\langle 0,\langle i, j\rangle\rangle}$ as follows: Wait until $\eta_{j}^{i}(0) \downarrow$; then search for $k, l$, with $k>0$ such that $\psi_{\langle k, l\rangle}(0) \downarrow=\eta_{j}^{i}(0)$. Then, let $\psi_{\langle 0,\langle i, j\rangle\rangle}=\psi_{\langle k, l\rangle}$. It is easy to verify that, if $\eta^{i}$ is a subnumbering of $\psi$ then $h(j)=\langle 0,\langle i, j\rangle\rangle$ reduces $\eta^{i}$ to $\psi$.

We let $\psi_{\langle i+1,0\rangle}(x)=\langle i, x\rangle$, for all $i, x$. The following construction will ensure that, for all $i$, for $e_{i}=\varphi_{i}(\langle i+1,0\rangle), \psi_{e_{i}}(\cdot) \neq \psi_{\langle i+1,0\rangle}\left(\left\langle e_{i}, \cdot\right\rangle\right)$. Thus ensuring that effective KRT for $\psi$ is not satisfied. In addition it will allow non-effective KRT. We now go on to define $\psi_{\langle k, l\rangle}$, for $k, l>0$. For the following we assume that pairing function is increasing in both the arguments (and thus also $\langle 0,0\rangle=0$ ).

Definition of $\psi_{\langle i+1, k\rangle}$ for $k>0$.

1. Dovetail steps 2 and 3 until wait in step 2 ends. If and when wait in step 2 ends, go to step 4.

2. Wait for $\varphi_{i}(\langle i+1,0\rangle)$ to converge (to say $z$ ), and then for $\psi_{z}(0)$ to converge.

3. For $\langle j, x\rangle=0$ to $\infty$

Let $\psi_{\langle i+1, j+1\rangle}(x)$ be same as $\psi_{\langle i+1, j\rangle}(\langle\langle i+1, j+1\rangle, x\rangle)$, whenever it gets defined.

4. If and when wait in step 2 ends, let $w=\psi_{z}(0)$. Let $n$ be such that, $n=w+j^{\prime}+3$, where $j^{\prime}$ is the maximum $j$ considered in the for-loop of step 3.

For $0<j<n, \psi_{\langle i+1, j\rangle}$ is no longer extended and therefore finite.

For all $x$, let $\psi_{\langle i+1, n\rangle}(x)=\psi_{\langle i+1,0\rangle}(\langle\langle i+1, n\rangle, x\rangle)$.

For $j=n$ to $\infty$ 
For all $x$, let $\psi_{\langle i+1, j+1\rangle}(x)=\psi_{\langle i+1, j\rangle}(\langle\langle i+1, j+1\rangle, x\rangle)$.

End definition of $\psi_{\langle i+1, \cdot\rangle}$.

Note that for any $i, \psi_{\langle i+1,0\rangle}(0)$ is of form $\langle i, 0\rangle$ and if $\psi_{\langle i+1, j+1\rangle} \neq \emptyset$, then $\psi_{\langle i+1, j+1\rangle}(0)$ is of the form $\langle i,\langle\langle i+1,1\rangle,\langle\langle i+1,2\rangle, \ldots,\langle\langle i+1, j+1\rangle, 0\rangle\rangle\rangle\rangle$ (in case step 2 does not succeed, or step 2 succeeds, but $j+1<n$ as in step 4) or of the form $\langle i,\langle\langle i+1, n\rangle,\langle\langle i+1, n+1\rangle, \ldots,\langle\langle i+1, j+1\rangle, 0\rangle\rangle\rangle\rangle$ (in case step 2 succeeds, and $j+1 \geq n$ as in step 4$)$.

If follows that, for any $i, j$ and $i^{\prime}, j^{\prime}, \psi_{\langle i+1, j\rangle}(0)=\psi_{\left\langle i^{\prime}+1, j^{\prime}\right\rangle}(0) \neq \uparrow$, then $i=i^{\prime}$ and $j=j^{\prime}$.

We now consider two cases:

Case 1: $\varphi_{i}(\langle i+1,0\rangle) \uparrow$ or $\varphi_{i}(\langle i+1,0\rangle) \downarrow=z$ and $\psi_{z}(0) \uparrow$.

In this case, clearly, $\varphi_{i}$ does not witness effectiveness of KRT. Also, $\langle i+$ $1, j+1\rangle$ acts as the $e$ in $\psi_{e}(x)=\psi_{\langle i+1, j\rangle}(\langle e, x\rangle)$.

Case 2: Not Case 1. In this case let $n, w$ be as in step 4 of the above construction.

Again, as in Case 1, it is easily seen that $\psi$ satisfies (non-effective) KRT. However, $z=\varphi_{i}(\langle i+1,0\rangle)$ does not act as $e$ for $\psi_{e}(x)=\psi_{\langle i+1,0\rangle}(\langle e, x\rangle)$. To see this, consider the following subcases:

Case 2.1: $\psi_{z}=\psi_{\left\langle i^{\prime}+1, j\right\rangle}$, for some $i^{\prime} \neq i$.

In this case, $\psi_{z}(0)=\psi_{\left\langle i^{\prime}+1, j\right\rangle}(0) \neq \psi_{\langle i+1,0\rangle}(\langle z, 0\rangle)$, since $\pi_{1}\left(\psi_{\left\langle i^{\prime}+1, j\right\rangle}(0)\right)=$ $i^{\prime} \neq i=\pi_{1}\left(\psi_{\langle i+1,0\rangle}(\langle z, 0\rangle)\right)$.

Case 2.2: $\psi_{z}=\psi_{\langle i+1, j\rangle}$, for some $j, 0<j<n$.

In this case, $\psi_{z}$ is finite, and thus $\psi_{z} \neq \lambda x \cdot \psi_{\langle i+1,0\rangle}(\langle z, x\rangle)$;

Case 2.3: $\psi_{z}=\psi_{\langle i+1,0\rangle}$.

In this case clearly, $\psi_{z} \neq \lambda x \cdot \psi_{\langle i+1,0\rangle}(\langle z, x\rangle)$.

On the other hand $\psi_{z}(0) \neq \psi_{\langle i+1, j\rangle}(0)$, for $j \geq n$, since, for $j \geq n$, $\psi_{\langle i+1, j\rangle}(0)=\langle i,\langle\langle i+1, n\rangle,\langle\langle i+1, n+1\rangle, \ldots,\langle\langle i+1, j\rangle, 0\rangle\rangle\rangle\rangle \geq n>\psi_{z}(0)$.

It follows from above subcases that $\psi_{z} \neq \lambda x \cdot \psi_{\langle i+1,0\rangle}(\langle z, x\rangle)$.

The set $U$ and the pairing functions defined in the proof of Theorem 15 can be used to show the following.

Theorem 21 There exists $U \subseteq \mathcal{P}$ and pairing functions $\langle\cdot, \cdot\rangle,\langle\langle\cdot, \cdot\rangle\rangle$, such that $U$ has $U$-acceptable numberings satisfying KRT when $\langle\cdot, \cdot\rangle$ is used, and $U$ has no $U$-acceptable numberings satisfying KRT when using $\langle\langle\cdot, \cdot\rangle\rangle$. 
We now consider the relationship between s-m-n-Theorem and KRT in $U$-complete numberings. We prove part (a) of the following theorem for $U$ acceptable numberings to simplify the proof, but this immediately yields the result for $U$-complete numberings as well.

\section{Theorem 22}

(a) There exists a $U$, and $U$-acceptable numbering $\psi$ such that $\psi$ satisfies effective KRT, but $\psi$ does not satisfy s-m-n-Theorem.

(b) For all $U$, for all $U$-complete numberings $\psi$, if $\psi$ satisfies the $s$-m-nTheorem, then $\psi$ satisfies effective KRT.

Proof. (a) We will use a similar approach as in the proof of Theorem 19. For the readers convenience, we will restate the assumptions we made about pairing and coding.

Again, we assume that pairing function is monotonically increasing in both its arguments (in particular $\langle 0,0\rangle=0$ ) and $\langle i, j\rangle>j$, for $\langle i, j\rangle \neq\langle 0,0\rangle$.

Let $\operatorname{code}(x)=x$. Further, for $n \geq 2$, we define $\operatorname{code}\left(x_{1}, x_{2}, \ldots, x_{n}\right)=$ $\left\langle x_{1}, \operatorname{code}\left(x_{2}, x_{3}, \ldots, x_{n}\right)\right\rangle$. We will need the following property of code. Each $z \in \mathbb{N}$ can be uniquely expressed as $z=\operatorname{code}\left(e_{1}, e_{2}, \ldots, e_{n}, 0\right)$, where $e_{n}>0$ (here $n$ may be 0 , i.e. $z=\operatorname{code}(0))$.

Let $f_{i}(x)=\langle i, x\rangle$. For $n \geq 1, g_{\left(e_{1}, e_{2}, \ldots, e_{n}\right)}^{i}(x)=f_{i}\left(\operatorname{code}\left(e_{1}, e_{2}, \ldots, e_{n}, x\right)\right)$. Note that

(A) $g_{\left(e_{1}\right)}^{i}(x)=f_{i}\left(\left\langle e_{1}, x\right\rangle\right)$, and $g_{\left(e_{1}, \ldots, e_{n}, e_{n+1}\right)}^{i}(x)=g_{\left(e_{1}, \ldots, e_{n}\right)}^{i}\left(\left\langle e_{n+1}, x\right\rangle\right)$.

We will be using $g_{\left(e_{1}, e_{2}, \ldots, e_{n}\right)}^{i}$ only for each $e_{j}$ being $\geq 1$.

We will define a computable numbering $\psi$ below. We let $U=\mathcal{P}_{\psi}$. It will be the case that $U=V$, where $V=\{\emptyset\} \cup\left\{f_{i} \mid i \in \mathbb{N}\right\} \cup\left\{g_{\left(e_{1}, e_{2}, \ldots, e_{n}\right)}^{i} \mid n \geq\right.$ $\left.1 \wedge(\forall j \in\{1, \ldots, n\})\left[e_{j} \geq 1\right]\right\}$.

Note that, $V$ above satisfies:

(B) for all $g, h \in V, g(0)=h(0)$ if and only if $g=h$. In particular, $f_{i}$ is the only function in $V$ which maps 0 to $\langle i, 0\rangle$, and, for $n>0$ and $\left.e_{1}, e_{2}, \ldots, e_{n}\right\rangle$ $0, g_{\left(e_{1}, e_{2}, \ldots, e_{n}\right)}^{i}$ is the only function in $V$ mapping 0 to $\left\langle i, \operatorname{code}\left(e_{1}, e_{2}, \ldots, e_{n}, 0\right)\right\rangle$.

We now define $\psi$.

(I) For all odd $i$, define $\psi_{\langle i, 0\rangle}$ to be equal to $f_{i}$.

(II) For all even $i$, define $\psi_{\langle i, 0\rangle}$ as follows.

Case 1: $\varphi_{i / 2}(0) \uparrow$. In this case $\psi_{\langle i, 0\rangle}$ is empty function.

Case 2: $\varphi_{i / 2}(0) \downarrow=\langle k, z\rangle$, and $z=0$. In this case let $\psi_{\langle i, 0\rangle}=f_{k}$. 
Case 3: $\varphi_{i / 2}(0) \downarrow=\langle k, z\rangle$, and $z>0$ and $z=\operatorname{code}\left(e_{1}, e_{2}, \ldots, e_{n}, 0\right)$, where each $e_{j}>0$. In this case let $\psi_{\langle i, 0\rangle}=g_{\left(e_{1}, e_{2}, \ldots, e_{n}\right)}^{k}$.

Case 4: $\varphi_{i / 2}(0) \downarrow=\langle k, z\rangle$, and $z>0$ and $z=\operatorname{code}\left(e_{1}, e_{2}, \ldots, e_{n}, 0\right)$, where $e_{n}>0$, and some $e_{j}=0, j \neq n$. In this case let $\psi_{\langle i, 0\rangle}=\emptyset$.

(III) For $j \geq 1$, let $\psi_{\langle i, j\rangle}$ be defined as follows.

Case 1: $\psi_{\langle i, j-1\rangle}(0) \uparrow$. In this case $\psi_{\langle i, j\rangle}$ is empty function.

Case 2: $\psi_{\langle i, j-1\rangle}(0) \downarrow=\langle k, z\rangle$, and $z=0$. In this case let $\psi_{\langle i, j\rangle}=g_{\left(e_{1}\right)}^{k}$, where $e_{1}=\langle i, j\rangle$.

Case 3: $\psi_{\langle i, j-1\rangle}(0) \downarrow=\langle k, z\rangle$, and $z>0$ and $z=\operatorname{code}\left(e_{1}, e_{2}, \ldots, e_{n}, 0\right)$, where each $e_{j}>0$. In this case let $\psi_{\langle i, j\rangle}=g_{\left(e_{1}, e_{2}, \ldots, e_{n}, e_{n+1}\right)}^{k}$, where $e_{n+1}=\langle i, j\rangle$.

Note that (by induction on $j$ ) the case of $\psi_{\langle i, j-1\rangle}(0) \downarrow=\langle k, z\rangle$, and $z>0$ and $z=\operatorname{code}\left(e_{1}, e_{2}, \ldots, e_{n}, 0\right)$, where $e_{n}>0$, and some $e_{j}=0, j \neq n$ cannot happen.

It is easy to verify that $\mathcal{P}_{\psi} \subseteq V$. (II) above ensures that (using property (B) above), if $\varphi_{i} \in V$, then $\psi_{\langle 2 i, 0\rangle}=\varphi_{i}$. (III) above ensures that effective KRT holds, since for all $i, j, x, \psi_{\langle i, j+1\rangle}(x)=\psi_{\langle i, j\rangle}(\langle\langle i, j+1\rangle, x\rangle)$ (see property (A) of $g_{\left(e_{1}, \ldots, e_{n}\right)}^{i}$ mentioned above).

We now claim that s-m-n-Theorem does not hold for $\psi$. This is so, since $\lambda x . f_{i}(\langle 0, x\rangle)$ does not belong to $V$; cf. the properties of code $(\cdot)$.

(b) Suppose $U \subseteq \mathcal{P}$ and $\psi$ is $U$-complete numbering which satisfies s-mn-Theorem. Suppose $s \in \mathcal{R}$ is such that, for all $i, j, x, \psi_{s(i, j)}(x)=\psi_{i}(\langle j, x\rangle)$. Define a function $u$ for all $i, j$ as follows.

$$
u(\langle i, j\rangle)= \begin{cases}s\left(i, \varphi_{j}(\langle i, j\rangle)\right), & \text { if } \varphi_{j}(\langle i, j\rangle) \downarrow ; \\ \uparrow, & \text { otherwise. }\end{cases}
$$

Let $\perp$ be the least element of $U$. Since $\psi$ is $U$-complete, there exists $r \in \mathcal{R}$, such that, for all $i, j$,

$$
\psi_{r(\langle i, j\rangle)}= \begin{cases}\psi_{u(\langle i, j\rangle)}, & \text { if } u(\langle i, j\rangle) \downarrow ; \\ \perp, & \text { otherwise. }\end{cases}
$$

Suppose $r=\varphi_{j}$ for some suitable $j$. Then, for all $i, \varphi_{j}(\langle i, j\rangle) \downarrow$. Thus, for all $i, x, \psi_{\varphi_{j}(\langle i, j\rangle)}(x)=\psi_{r(\langle i, j\rangle)}(x)=\psi_{s\left(i, \varphi_{j}(\langle i, j\rangle)\right)}(x)=\psi_{i}\left(\left\langle\varphi_{j}(\langle i, j\rangle), x\right\rangle\right)$. Thus, $\lambda i . \varphi_{j}(\langle i, j\rangle)$ witnesses effective KRT for $\psi$.

The next result will show that the assumption " $\psi U$-complete" cannot be dropped from Theorem 22(b).

Theorem 23 There exists $U \subseteq \mathcal{P}$ with least element and a numbering $\psi$ for $U$ that satisfies effective $s-m$-n-Theorem, but not KRT. 
Proof. Let $f(x)=x$. Let $d$ be a computable isomorphism from non-empty finite sequences over $\mathbb{N}$ to $\{2,3,4, \ldots\}$, such that, for all $e, d((e)) \neq e$. Clearly, there exists such a mapping.

Define $\psi$ as follows.

$\psi_{0}=\emptyset . \psi_{1}=f . \psi_{d\left(\left(e_{1}, e_{2}, \ldots, e_{k}\right)\right)}(x)=f\left(\left\langle e_{1},\left\langle e_{2}, \ldots,\left\langle e_{k}, x\right\rangle\right\rangle\right\rangle\right)$.

It is easy to verify that $\psi_{d\left(\left(e_{1}, e_{2}, \ldots, e_{k}, e_{k+1}\right)\right)}(x)=\psi_{d\left(\left(e_{1}, e_{2}, \ldots, e_{k}\right)\right)}\left(\left\langle e_{k+1}, x\right\rangle\right)$ holds for $k \geq 1$. Moreover, $\psi_{d((e))}(x)=\psi_{1}(\langle e, x\rangle)$, and $\psi_{0}(x)=\psi_{0}(\langle e, x\rangle)$, for all $e$.

Let

$$
s(m, n)= \begin{cases}0, & \text { if } m=0 \\ d((n)), & \text { if } m=1 ; \\ d\left(\left(e_{1}, e_{2}, \ldots, e_{k}, n\right)\right), & \text { if } 1<m=d\left(e_{1}, \ldots, e_{k}\right)\end{cases}
$$

It is easy to verify that $s(m, n)$ witnesses the s-m-n-Theorem for $\psi$. $\psi$ does not satisfy KRT, since for all $e, \psi_{e} \neq \lambda x . f(\langle e, x\rangle)$ (note that $d((n)) \neq$ $n)$.

The following theorem proves that composition and KRT are independent in complete numberings.

Theorem 24 There exist $U, V \subseteq \mathcal{P}$ such that

(a) there exists a $U$-acceptable numbering with effective composition,

(b) there exists no U-complete numbering satisfying (even non-effective) KRT;

(c) there exists a $V$-acceptable numbering satisfying $K R T$,

(d) there exists no $V$-complete numbering with (even non-effective) composition.

Proof. Let $U=\{\emptyset, i d\}$. As in the proof of Theorem 16, every $U$-complete numbering has composition. Furthermore, the proof of Theorem 18 shows, that no $U$-complete numberings satisfies KRT; yielding (a) and (b).

Parts (c) and (d) follow immediately from Theorems 17 and 22(b).

In [11] the following criterion for recursively enumerable index sets in acceptable numberings is proved; originally due to [14].

Recall that $F_{0}, F_{1}, \ldots$ denotes the canonical indexing of finite functions. 
Theorem 25 ([11]) Suppose $U \subseteq \mathcal{P}$ and $\psi$ is an acceptable numbering. Then, $I_{U}^{\psi}$ is recursively enumerable if and only if there exists an recursively enumerable set $A$ such that

$$
I_{U}^{\psi}=\left\{x \mid(\exists y \in A)\left[F_{y} \subseteq \psi_{x}\right]\right\} .
$$

This nice property does not hold for $U$-acceptable numberings. Hence, it can not hold for complete numberings either.

Theorem 26 There exists $U \subseteq \mathcal{P}$, a $U$-acceptable numbering $\psi$ and a set $V \subseteq U$ such that

(a) $I_{V}^{\psi}$ is recursively enumerable, and

(b) for all numberings $\eta$ such that $I_{V}^{\psi}=\left\{i \mid(\exists j)\left[\eta_{j} \subseteq \psi_{i}\right]\right\}$, there exists $k$ such that $\eta_{k}$ has infinite domain.

This result is quite surprising. If $I_{V}^{\psi}$ is recursively enumerable, this means that for every $i$ such that $i$ is enumerated in $I_{V}^{\psi}$, only a finite part of $\psi_{i}$ may have been computed before enumerating $i$, and this finite part was sufficient to recognize $\psi_{i}$ as a member of $V$. However, part (b) indicates that this finite part must depend on index $i$, and not just the function computed by $\psi_{i}$, despite the fact that $I_{V}^{\psi}$ itself depends only on the functions computed by the indices.

Proof. Let $\eta^{0}, \eta^{1}, \ldots$ denote an recursively enumerable sequence of all the numberings. We will define a numbering $\psi$ below. Let $U=\left\{\psi_{k} \mid k \in \mathbb{N}\right\}$, and $V=\left\{\psi_{\langle i+1,0\rangle} \mid i \in \mathbb{N}\right\}$. Let, $I_{i}=\left\{j \mid \psi_{j}=\psi_{\langle i+1,0\rangle}\right\}$. Thus, $I_{V}^{\psi}=\bigcup_{i \geq 0} I_{i}$ is the index set for $V$.

If $\varphi_{k}(0) \uparrow$, then $\psi_{\langle 0, k\rangle}$ is defined to be the empty function. In the following construction we will define, effectively in $i$,

(I) $\psi_{\langle i+1, j\rangle}, j \in \mathbb{N}$,

(II) $\psi_{\langle 0, j\rangle}$, such that $\varphi_{j}(0)=i$, and

(III) $I_{i}$.

Note that the above covers the definition of all $\psi_{k}$.

The construction will satisfy the following properties for each $i$.

(A) $I_{i}=\left\{j \mid \psi_{j}=\psi_{\langle i+1,0\rangle}\right\}$, is recursively enumerable (where an index for enumerating $I_{i}$ can be found effectively in $i$ ).

(B) If $\varphi_{k}(0)=i$ and $\varphi_{k} \in U$, then $\psi_{\langle 0, k\rangle}=\varphi_{k}$. 
(C) If there exists a $k$ such that $\eta_{k}^{i}$ is finite and range $\left(\eta_{k}^{i}\right) \subseteq\{i\}$, then, for some $j^{\prime}, k^{\prime}, \eta_{j^{\prime}}^{i} \subseteq \psi_{\left\langle i+1, k^{\prime}\right\rangle}$, but $\psi_{\left\langle i+1, k^{\prime}\right\rangle} \notin V$.

(D) For all $j$, range $\left(\psi_{\langle i+1, j\rangle}\right) \subseteq\{i\}$. For all $j$, such that $\varphi_{j}(0)=i$, range $\left(\psi_{\langle 0, j\rangle}\right) \subseteq\{i\}$. Moreover, either $\psi_{\langle i+1, j\rangle}=\emptyset$ or $\psi_{\langle i+1, j\rangle}(0)=i$. Similarly, for $j$ such that $\varphi_{j}(0)=i$, either $\psi_{\langle 0, j\rangle}=\emptyset$ or $\psi_{\langle 0, j\rangle}(0)=i$.

Note that the above properties will prove the theorem: (B) along with the fact that, if $\varphi_{k}(0) \uparrow$ and $\varphi_{k} \in U$, then $\varphi_{k}=\psi_{\langle 0, k\rangle}=\emptyset$, shows that $\psi$ is acceptable for $U$; (A) shows that $I_{V}^{\psi}$ is recursively enumerable; (C) and ( D) show that part (b) of the theorem holds. Property (D) is mainly used for ease of understanding and avoiding interference between the construction for different $i$.

We now proceed with the construction, for a given $i$, of (I) $\psi_{\langle i+1, j\rangle}, j \in \mathbb{N}$, (II) $\psi_{\langle 0, j\rangle}$, such that $\varphi_{j}(0)=i$, and (III) $I_{i}$.

Let $a_{i}^{0}, a_{i}^{1}, \ldots$ be recursive sequence of numbers satisfying the following: if there exists a $j$ such that range $\left(\eta_{j}^{i}\right) \subseteq\{i\}$ and domain $\left(\eta_{j}^{i}\right)$ is finite, then, for one such $j, \lim _{s \rightarrow \infty} a_{i}^{s} \downarrow \geq \max \left(\operatorname{domain}\left(\eta_{j}^{i}\right)\right)$. On the other hand, if there does not exists a $j$ such that range $\left(\eta_{j}^{i}\right) \subseteq\{i\}$ and $\eta_{j}^{i}$ is finite, then $\lim _{s \rightarrow \infty} a_{i}^{s \uparrow}$. Note that such a recursive sequence can easily be constructed (effectively from $i$ ). We further assume without loss of generality that $a_{i}^{s} \geq 2$, for all $i, s$.

Initially, let $\psi_{\langle i+1,0\rangle}(0)=i$. Let $t_{i}^{0}=0$, and $Q_{i}^{0}=\emptyset$. Intuitively, $t_{i}^{s}$ denotes the maximum $j$ such that $\langle i+1, j\rangle$ has been used for diagonalization up to (start of) stage $s$ in the construction. $Q_{i}^{s}$ denotes the set of programs $j$, such that it has been detected up to (start of) stage $s$ that $\varphi_{j}(0)=i$. Let $\psi_{k}^{s}$ denote $\psi_{k}$ defined before the start of stage $s$. Initially $\langle i+1,0\rangle$ is enumerated in $I_{i}$.

Definition of $I_{i} ; \psi_{\langle i+1, j\rangle}, j \in \mathbb{N}$; and $\psi_{\langle 0, k\rangle}$, such that $\varphi_{k}(0)=i$.

Go to stage 0.

Stage $s$

1. Let $m=\max \left(\left\{a_{i}^{s}\right\} \cup \operatorname{domain}\left(\psi_{\langle i+1,0\rangle}^{s}\right)\right)$.

Let $r=\operatorname{card}\left(Q_{i}^{s}\right)$.

2. For $j \leq t_{i}^{s}$, and $x \leq m+r+2$, let $\psi_{\langle i+1, j\rangle}(x)=i$.

For $j \in Q_{i}^{s}$, and $x \leq m+r+2$, let $\psi_{\langle 0, j\rangle}(x)=i$.

3. For $j$, such that $0<j \leq r+2$, and $x \leq m$, let $\psi_{\left\langle i+1, t_{i}^{s}+j\right\rangle}(x)=i$.

For $j$, such that $0<j \leq r+2$, let $\psi_{\left\langle i+1, t_{i}^{s}+j\right\rangle}(m+j)=i$. 
(* Note that $\psi_{\langle i+1,0\rangle}$ has all of $X=\{x \mid m<x \leq m+r+2\}$ in its domain. However, for $0<j \leq r+2, \psi_{\left\langle i+1, t_{i}^{s}+j\right\rangle}$ has $m+j$ but not $X \backslash\{m+j\}$ in its domain so far. $\left.{ }^{*}\right)$

4. Dovetail steps 5, 6 and 7, until step 5 succeeds. If and when step 5 succeeds, go to step 8.

5. Search for $s^{\prime}>s$, such that $a_{i}^{s} \neq a_{i}^{s^{\prime}}$.

6. For $z=0$ to $\infty$ do

For $k \in Q_{i}^{s}$, if $\left\{x \mid m<x \leq m+r+2 \wedge \Phi_{k}(x) \leq z\right\}=\{m+j\}$, where $0<j \leq r+2$, then, for $w \leq m+r+2$, let $\psi_{\left\langle i+1, t_{i}^{s}+j\right\rangle}(w)=i$ and enumerate $\left\langle i+1, t_{i}^{s}+j\right\rangle$ in $I_{i}$.

(Note that $\psi_{\left\langle i+1, t_{i}^{s}+j\right\rangle}$ is made equal to $\psi_{\langle i+1,0\rangle}$ in this case).

End For

(* The above step makes those $\psi_{\left\langle i+1, t_{i}^{s}+j\right\rangle}$ to be equal to $\psi_{\langle i+1,0\rangle}$, which seem to be equal to some $\varphi_{k}, k \in Q_{i}^{s}$. This is needed to make sure that the reduction from $\varphi_{k} \rightarrow \psi_{\langle 0, k\rangle}$ works. $\left.{ }^{*}\right)$

7. For $z=0$ to $\infty$

For $k \leq z$, such that $k \notin Q_{i}^{s}, \Phi_{k}(0) \leq z$, and $\varphi_{k}(0) \downarrow=i$ :

(a) If $\operatorname{card}\left(\operatorname{domain}\left(\varphi_{k}\right) \cap\{x \mid m<x \leq m+r+2\}\right) \geq 2$, then enumerate $\langle 0, k\rangle$ in $I_{i}$, and for $x \leq m+r+2$, let $\psi_{\langle 0, k\rangle}(x)=i$.

(b) If domain $\left(\varphi_{k, z}\right) \cap\{x \mid m<x \leq m+r+2\}=\{m+j\}$, then for $x \leq m+r+2$, such that $\psi_{\left\langle i+1, t_{i}^{s}+j\right\rangle}(x)$ is defined up to now, let $\psi_{\langle 0, k\rangle}(x)=i$; additionally enumerate $\langle 0, k\rangle \in I_{i}$, if $\left\langle i+1, t_{i}^{s}+j\right\rangle$ has been enumerated in $I_{i}$.

End For

( ${ }^{*}$ The above step is essentially doing the reduction $\varphi_{k} \rightarrow \psi_{\langle 0, k\rangle}$, for $k$ such that $\varphi_{k}(0)=i$, but $k \notin Q_{i}^{s}$. $\left.{ }^{*}\right)$

8. Let $Q_{i}^{s+1}$ denote all the $k$ 's such that $k \in Q_{i}^{s}$ or $\left[\Phi_{k}(0) \leq s\right.$ and $\left.\varphi_{k}(0) \downarrow=i\right]$ or $\varphi_{k}(0) \downarrow=i$ has been discovered in step 7 in this stage.

Let $t_{i}^{s+1}=t_{i}^{s}+r+2$.

Enumerate $\langle i+1, j\rangle$ such that $j \leq t_{i}^{s+1}$ in $I_{i}$.

Enumerate $\langle 0, j\rangle$ such that $j \in Q_{i}^{s+1}$ in $I_{i}$.

Go to stage $s+1$.

End Stage $s$ 
Any $\psi_{\langle i+1, w\rangle}$ not defined by above procedure is empty. Note that (D) is trivially satisfied by construction. We now show that (A), (B) and (C) hold for each $i$. Fix an $i$. Let $Q_{i}=\left\{j \mid \varphi_{j}(0)=i\right\}$. Consider the following cases in the construction (for the fixed $i$ ).

Case 1: There exist infinitely many stages in the construction.

In this case, for all $j, \psi_{\langle i+1, j\rangle}$ is the constant $i$ function. Also, for all $j \in Q_{i}, \psi_{\langle 0, j\rangle}$ is also the constant $i$ function. Thus, (B) is satisfied. Note that $I_{i}=\left\{\langle 0, j\rangle \mid j \in Q_{i}\right\} \cup\{\langle i+1, j\rangle \mid j \in \mathbb{N}\}$ (step 8). Thus, (A) is satisfied. Also, $\lim _{s \rightarrow \infty} a_{i}^{s}$ diverges. Thus, for all $j$, either range $\left(\eta_{j}^{i}\right) \nsubseteq\{i\}$ or $\eta_{j}^{i}$ has infinite domain. Thus $(\mathrm{C})$ is satisfied.

Case 2: Stage $s$ starts but does not finish.

In this case, for all $s^{\prime} \geq s, a_{i}^{s}=a_{i}^{s^{\prime}}$, and thus there exists a $j$ such that $\eta_{j}^{i} \subseteq\left\{(x, i) \mid x \leq a_{i}^{s}\right\}$. Let $m, r, t_{i}^{s}, Q_{i}^{s}$ be as in stage $s$. Note that,

(i) for $j \in Q_{i}^{s}, \psi_{\langle 0, j\rangle}=\psi_{\langle i+1,0\rangle}=\{(x, i) \mid x \leq m+r+2\}$;

(ii) for $j \leq t_{i}^{s}, \psi_{\langle i+1, j\rangle}=\psi_{\langle i+1,0\rangle}=\{(x, i) \mid x \leq m+r+2\}$;

(iii) for $j>t_{i}^{s}+r+2, \psi_{\langle i+1, j\rangle}=\emptyset$;

(iv) for $0<j \leq r+2$, either $\psi_{\left\langle i+1, t_{i}^{s}+j\right\rangle}=\psi_{\langle i+1,0\rangle}$ holds, or it holds that $\operatorname{domain}\left(\psi_{\left\langle i+1, t_{i}^{s}+j\right\rangle}\right)=\{x \mid x \leq m\} \cup\{m+j\}$;

(v) For $k \in Q_{i}^{s}$, if $\operatorname{domain}\left(\varphi_{k}\right) \cap\{m+x \mid 0<x \leq r+2\}=\{m+j\}$, then $\psi_{\left\langle i+1, t_{i}^{s}+j\right\rangle}=\psi_{\langle i+1,0\rangle}$, and $\psi_{\langle i+1,0\rangle}$ is the only function in $U$ with range $\{i\}$, and containing $m+j$ in the domain;

(vi) For $k \in Q_{i} \backslash Q_{i}^{s}$, if $\operatorname{domain}\left(\varphi_{k}\right) \cap\{m+x \mid 0<x \leq r+2\}=\{m+j\}$, then $\psi_{\left\langle i+1, t_{i}^{s}+j\right\rangle}=\psi_{\langle 0, k\rangle}$, moreover, $\psi_{\left\langle i+1, t_{i}^{s}+j\right\rangle}$ is the only function (if any) in $U$ which contains $\{(0, i)\}$ and is defined on $m+j$ but not on $m+j^{\prime}$, $0<j^{\prime} \leq r+2, j^{\prime} \neq j$

(vii) For $k \in Q_{i} \backslash Q_{i}^{s}$, if $\operatorname{card}\left(\operatorname{domain}\left(\varphi_{k}\right) \cap\{m+x \mid 0<x \leq r+2\}\right) \geq 2$, then $\psi_{\langle i+1,0\rangle}=\psi_{\langle 0, k\rangle}$, moreover, $\psi_{\langle i+1,0\rangle}$ is the only function (if any) in $U$ which contains $\{(0, i)\}$ and is defined on at least two inputs of the form, $m+j^{\prime}, 0<j^{\prime} \leq r+2$.

From the above it immediately follows that (B) holds. (A) can also be seen to hold, since any time $\psi_{k}$ contains at least two of $\{m+j \mid 0<j \leq r+2\}$, in its domain, $k$ is enumerated in $I_{i}$ (note that $\psi_{\langle i+1,0\rangle}$ is the only function in $U$ which contains at least two of $\{m+j \mid 0<j \leq r+2\}$ in its domain). To see (C), first note that at least one of $\psi_{\left\langle i+1, t_{i}^{s}+j\right\rangle}, 0<j \leq r+2$, is not equal to $\psi_{\langle i+1,0\rangle}$ (since cardinality of $Q_{i}^{s}$ is $r$, and thus at most $r$ of $\psi_{\left\langle i+1, t_{i}^{s}+j\right\rangle}$, $0<j \leq r+2$, can be made equal to $\psi_{\langle i+1,0\rangle}$ due to step 6). However, $\left\{(x, i) \mid x \leq a_{i}^{s}\right\}$ is contained in all $\psi_{\left\langle i+1, t_{i}^{s}+j\right\rangle}, 0<j \leq r+2$. Thus (C) is satisfied. This proves the theorem. 
The next two results could be interpreted as a warning: one has to be rather careful in carrying over properties from acceptable numberings to $U$ complete numberings.

\section{Theorem 27}

(a) For any acceptable numbering $\psi$ and any $f \in \mathcal{P}, I_{f}^{\psi}$ is not recursively enumerable.

(b) There exists $U \subseteq \mathcal{P}$, $\operatorname{card}(U)>1$, such that $U$ has a $U$-acceptable numbering, and there exists an $f \in U$ such that, for any $U$-complete numbering $\psi, I_{f}^{\psi}$ is recursively enumerable.

Proof. (a) See, for example, [7].

(b) Let $g$ be the everywhere 0 function. Let $U=\{\emptyset, g\}$. $U$ has a $U$ acceptable numbering by Proposition 1. Moreover, $\left\{i \mid \psi_{i}=g\right\}$ is obviously recursively enumerable for every $U$-complete numbering $\psi$.

For any acceptable numbering $\psi$, the general halting problem - the set $\left\{(x, y) \mid \psi_{x}(y) \downarrow\right\}$ - and the special halting problem - the set $\left\{x \mid \psi_{x}(x) \downarrow\right\}-$ are essentially the same. This no longer holds for $U$-complete numberings.

\section{Theorem 28}

(a) If $U \subseteq \mathcal{P}, \operatorname{card}(U)>1$ and $\psi$ is $U$-complete, then $K_{\psi}=\{(x, y) \mid$ $\left.\psi_{x}(y) \downarrow\right\}$ is not recursive.

(b) There exists $U \subseteq \mathcal{P}$, $\operatorname{card}(U)>1$, having a $U$-complete numbering $\psi$ such that $\left\{x \mid \psi_{x}(x) \downarrow\right\}$ is recursive.

Proof. (a) Choose $U$ and $\psi$ as in the hypotheses. Suppose by way of contradiction, that $K_{\psi}$ is recursive. Suppose $\perp$ is the least element of $U$ and let $\psi_{j} \in U$ be such that $\perp \neq \psi_{j}$ (note that $\operatorname{card}(U)>1$ ). Let $A$ be any recursively enumerable but not recursive set. For all $i$, define

$$
u(i)= \begin{cases}j, & \text { if } i \in A \\ \uparrow, & \text { otherwise }\end{cases}
$$

Clearly, $u$ is partial recursive. Since $\psi$ is $U$-complete, there exists $r \in \mathcal{R}$ such that, for all $i$,

$$
\psi_{r(i)}= \begin{cases}\psi_{j}, & \text { if } i \in A \\ \perp, & \text { otherwise }\end{cases}
$$


Let $n$ be such that $\psi_{j}(n) \downarrow$ but $\perp(n) \uparrow$ (note that there exists such an $n$ since $\left.\perp \subset \psi_{j}\right)$. Now, define

$$
g(i)= \begin{cases}1, & \text { if } \psi_{r(i)}(n) \downarrow \\ 0, & \text { otherwise }\end{cases}
$$

So, $g(i)=1$ if and only if $\psi_{r(i)}(n) \downarrow$ if and only if $i \in A$. Thus $g$ is a characteristic function of $A$. Since $K_{\psi}$ is recursive by assumption, $g$ is a recursive function. But then $A$ is recursive, a contradiction.

(b) Let $f$ be such that $f(0)=1$, and $f(x) \uparrow$ for $x>0$. Let $U=\{\emptyset, f\}$. Let $\eta$ be a $U$-complete numbering (clearly, there exists such a $U$-complete numbering). Let $\psi_{0}=\emptyset$, and $\psi_{i+1}=\eta_{i}$. Thus, $\psi$ is $U$-complete. However, for all $x, \psi_{x}(x) \uparrow$, and thus $\left\{x \mid \psi_{x}(x) \downarrow\right\}=\emptyset$ is recursive.

\section{References}

[1] Angluin, D. (1980), Inductive Inference of Formal Languages from Positive Data, Information and Control 45, 117 - 135.

[2] Blum, M. (1967), A Machine Independent Theory of the Complexity of Recursive Functions, Journal of the ACM, 14, 322-336.

[3] Case, J., Jain, S., Suraj, M. (1997), Control Structures in Hypothesis Spaces: The Influence on Learning, In S. Ben-David, Ed., Third European Conference on Computational Learning Theory, 286 - 300, Lecture Notes in Artificial Intelligence 1208, Springer-Verlag, 1997.

[4] Eršov, Yu. L. (1973), Theorie der Numerierungen 1, Zeitschrift für Mathematische Logik und Grundlagen der Mathematik 19, 289 - 388.

[5] Freivalds, R., Kinber, E., Wiehagen, R. (1982), Inductive inference and computable one-one numberings, Zeitschrift für Mathematische Logik und Grundlagen der Mathematik 28, 463 - 479.

[6] Freivalds, R., Kinber, E., Wiehagen, R. (1995), How Inductive Inference Strategies Discover Their Errors, Information and Control 118, 208 226. 
[7] Hay, L. (1966), Isomorphism types for index sets of partial recursive functions, Proceedings of the American Mathematical Society 16, 106 110.

[8] Kummer, M. (1995), A learning-theoretic characterization of classes of recursive functions, Information Processing Letters 54, 205 - 211.

[9] Lange, S., Zeugmann, T. (1993), Language learning in dependence of the space of hypotheses, In L. Pitt, Ed., Proceedings 6th Annual ACM Conference on Computational Learning Theory, 127 - 136, ACM Press, New York.

[10] Lange, S., Zeugmann, T. (1994), Characterization of Language Learning under Various Monotonicity Constraints, Journal of Experimental and Theoretical Artificial Intelligence 6, 73 - 94.

[11] Machtey, M., Young, P. (1978), An Introduction to the General Theory of Algorithms, North Holland, New York.

[12] Mal'cev, A. I. (1970), Algorithms and recursive functions, WolthersNoordhoff Publishing, Groningen.

[13] Riccardi, G. (1980), The Independence of Control Structures in Abstract Programming Systems, PhD Thesis, State University of New York at Buffalo.

[14] Rice, H. G. (1956), On completely recursively enumerable classes and their key arrays, The Journal of Symbolic Logic 21, 304 - 308.

[15] Rogers, H. Jr. (1987), Theory of Recursive Functions and Effective Computability, MIT Press, Cambridge, Massachusetts.

[16] Royer, J. S. (1987), A Connotational Theory of Program Structure, Lecture Notes in Computer Science 273, Springer-Verlag, Berlin. 